\title{
THE EXTENDED MODULE OF INDECOMPOSABLES FOR $\bmod 2$ FINITE $H$-SPACES
}

BY

\author{
R. M. KANE ${ }^{1}$
}

\begin{abstract}
We construct a generalization of the usual module of indecomposables for the $\bmod 2$ cohomology of a finite $H$-space. Structure theorems are obtained regarding how the Steenrod algebra acts on this module.
\end{abstract}

1. Introduction. Let $H^{*}=H^{*}(X ; \mathbf{Z} / 2)$, where $\mathbf{Z} / 2$ are the integers reduced $\bmod 2$. Throughout this paper $(X, \mu)$ will be a mod 2 finite $H$-space. A great deal of work has been devoted to the study of the module of indecomposables $Q=Q H^{*}$ of such spaces. The most recent related work has been centred around proving the loop space conjective that $H^{*}(\Omega X ; \mathbf{Z})$ has no 2 torsion when $X$ is simply connected. It was shown in [4] that the absence of 2 torsion in $H^{*}(\Omega X ; \mathbf{Z})$ is equivalent to the triviality of $Q^{\text {even }}$. Moreover, it was shown in [5] that $Q^{4 n}=0$ for $n \geqslant 1$. The triviality of $Q^{\text {even }}$ in the remaining degrees has proved more elusive. In [8-11] Lin has proved that $Q^{\text {even }}=0$ provided $H_{*}$ is associated. In [6] we hope to present an alternative proof of the triviality of $Q^{\text {even }}$. Moreover, the associativity hypothesis will not be required. The proof uses the machinery of Morava $K$-theory. Before we can apply Morava $K$-theory, however, some knowledge of the Steenrod module structure of $Q$ is required. That is the purpose of this paper.

Actually we will work with a more general structure than $Q$. Let

$$
Q_{n} H^{*}=\hat{H}^{*} / D^{n+1},
$$

where $D^{n+1}$ are the $n+1$ fold decomposables of $H^{*}$. In particular, $Q=Q_{1} H^{*}$. The reduced coproduct $\bar{\mu}^{*}: H^{*} \rightarrow H^{*} \otimes H^{*}\left(\bar{\mu}^{*} x=\mu^{*} x-x \otimes 1-1 \otimes x\right)$ induces maps

$$
\bar{\mu}^{*}: Q_{s+t} H^{*} \rightarrow Q_{s} H^{*} \otimes Q_{t} H^{*}
$$

for any $s, t \geqslant 1$. We will use the map $\bar{\mu}^{*}: Q_{2} H^{*} \rightarrow Q \otimes Q$. Define $\hat{Q} \subset Q_{2} H^{*}$ by

$$
\hat{Q}=\operatorname{ker} \bar{\mu}^{*} Q_{2} H^{*} \rightarrow Q \otimes Q .
$$

We will study $\hat{Q}^{\text {even }}$. Let $\mathscr{A}(2)$ be the mod 2 Steenrod algebra. There is an action of $\mathscr{A}(2)$ on $\hat{Q}^{\text {even }}$. First of all, the action of $\mathscr{A}(2)$ on $H^{*}$ induces an action on $Q_{2} H^{*}$. By naturality there is an action of $\mathscr{A}(2)$ on $\hat{Q}$. In $\S 2$ we will show that $\mathrm{Sq}^{1} \hat{Q}^{\text {even }}=0$. So $\mathscr{A}(2)$ leaves $\hat{Q}^{\text {even }}$ invariant. In this paper we will study the Steenrod module structure of $\hat{Q}^{\text {even }}$.

Received by the editors February 10, 1984 and, in revised form, January 24, 1985.

1980 Mathematics Subject Classification. Primary 57T25; Secondary 55S20, 57T05.

Key words and phrases. $H$-space, mod 2 finite, indecomposables, primitive, Steenrod algebra, secondary operation.

${ }^{1}$ Research partially supported by NSERC Grant \# A4853.

(01985 American Mathematical Society $0002-9947 / 85 \$ 1.00+\$ .25$ per page 
Any information about $\hat{Q}^{\text {even }}$ also gives information about $Q^{\text {even }}$. The relation of $\hat{Q}^{\text {even }}$ to $Q^{\text {even }}$ is determined by the following exact sequence. Consider the Frobenius squaring map

$$
\xi: H^{*} \rightarrow H^{*}, \quad \xi(x)=x^{2} .
$$

Then $\xi H^{*}$ is a sub-Hopf algebra. We will see in $\$ 2$ that we have a short exact sequence

$$
0 \rightarrow Q \xi H^{*} \rightarrow \hat{Q}^{\text {even }} \rightarrow Q^{\text {even }} \rightarrow 0
$$

of Steenrod modules. Because of (1.1) we call $\hat{Q}$ the extended module of indecomposables. Working with $\hat{Q}^{\text {even }}$ rather than $Q^{\text {even }}$ is roughly equivalent to working with a simple system of generators for $H^{*}$. Our structure theorems for $\hat{Q}^{\text {even }}$ tell how even degree simple generators are tied together via Steenrod operations.

Our results are modelled on those obtained by Thomas in [14 and 15]. In those papers he worked under the hypothesis that $H^{*}$ is primitively generated and obtained powerful structure theorems for the action of $\mathscr{A}(2)$ on $Q$. Given $n \geqslant 0$ let $n=\sum_{s \geqslant 0} n_{s} 2^{s}$ be its binary expansion. We will say that $2^{s} \in n$ if $n_{s} \neq 0$ and $2^{s} \notin n$ if $n_{s}=0$. Thomas proved

$$
\mathrm{Sq}^{2^{s}} Q^{n}=0 \quad \text { if } 2^{s} \notin n, \quad Q^{n}=\mathrm{Sq}^{2^{s}} Q^{n-2^{s}} \quad \text { if } 2^{s} \notin n \text { and } 2^{s}<n .
$$

We will prove the following weaker version.

THEOREM 1.2. Let $(X, \mu)$ be $a$ mod 2 finite $H$-space and $s \geqslant 1$.

(a) If $2^{s} \notin 2 n$, then $\mathrm{Sq}^{2^{s}} \hat{Q}^{2 n}=0$.

(b) If $2 s \notin 2 n$ and $2^{s+1} \in 2 n$, then $\hat{Q}^{2 n}=\mathrm{Sq}^{2^{s}} \hat{Q}^{n-2^{s}}$.

It is a simple consequence of $1.2(\mathrm{~b})$ that

COROLlary 1.3. Let $(X, \mu)$ be a $\bmod 2$ finite $H$-space. Then $\hat{Q}^{\text {even }}$ is generated on $\mathscr{A}(2)$ by $\sum_{i \geqslant 2} \hat{Q}^{2^{\prime}-2}$.

Actually, we will prove 1.3 in the process of proving 1.2 (see $\S 5$ ). So it is probably more accurate to call 1.2 a consequence of 1.3 .

It should be noted that the above results apply to $Q^{\text {even }}=\hat{Q}^{\text {even }} / Q \xi H^{*}$. However, it does not seem possible to prove Theorem 1.2 for $Q^{\text {even }}$ separately. Our arguments force us to work with the more general structure of $\hat{Q}^{\text {even }}$. Also, we will require a knowledge of $\hat{Q}^{\text {even }}$, not just $Q^{\text {even }}$, for the arguments in [6].

This paper has seven more sections. In $\S 2$ we establish some facts about $H^{*}$ and $H_{*}$. In $\S \S 3-6$ we use secondary operations to prove results about the action of $\mathscr{A}(2)$ on $\hat{Q}^{\text {even }}$. Our results and proofs are modelled on those of Lin in [8]. In $\S \S 7$ and 8 we then use these results to prove Theorem 1.2. The main new idea in our proof is the systematic use of Wall's description of $\mathscr{A}(2)$ in [16].

Throughout this paper $(X, \mu)$ will be a mod 2 finite $H$-space. By a $H$-space we mean a pointed topological space $X$ having the homotopy type of a connected $\mathrm{CW}$ complex of finite type together with a base-point preserving map $\mu: X \times X \rightarrow X$ with two sided homotopy unit. An $H$-space is $\bmod 2$ finite if $H^{*}$ is finite. We will be assuming a knowledge of Hopf algebra theory. We refer the reader to [13] for details. 
This paper is a revised and greatly expanded version of a previous paper. I would like to thank the referee of that previous version for pointing out an error in my proof of property (1.1).

2. The module of indecomposables. We begin with some facts about the mod 2 homology and cohomology of mod 2 finite $H$-spaces. There is a duality between $H^{*}$ and $H_{*}$ as Hopf algebras over $\mathscr{A}(2)$. This induces a duality between $Q=Q H^{*}$ and $P=P H_{*}$, the module of primitives. The action of $\mathscr{A}(2)$ on $H_{*}$, and on $P$, is the right action obtained by duality from the left action of $\mathscr{A}(2)$ on $H^{*}$ and on $Q$. So $\langle\alpha \theta, x\rangle=\langle\alpha, \theta x\rangle$ for any $\alpha \in H_{*}, x \in H^{*}$ and $\theta \in \mathscr{A}(2)$. In $Q$ we have the restrictions

LEMMA 2.1. $Q^{4 n}=0$ for $n \geqslant 1$.

LEMMA 2.2. $\mathrm{Sq}^{1}=0$ on $Q^{\text {even }}$.

In $P$ we have the following restrictions:

Lemma 2.3. For any $\alpha, \beta \in P_{\text {odd }}, \alpha^{2}=\beta^{2}=0$ and $\alpha \beta=\beta \alpha$.

LeMmA 2.4. For any $\alpha, \beta \in P_{\text {even }}, \alpha^{2}=\beta^{2}=0$ and $\alpha \beta=\beta \alpha$.

Lemma 2.5. $\mathrm{Sq}^{1}=0$ on $P_{\text {odd }}$.

For all of the above facts consult [5]. We now use the above facts plus some Hopf algebra arguments to demonstrate that the module $\hat{Q}^{\text {even }}$ described in $\S 1$ satisfies the properties stated there. The exactness of (1.1) follows from the next two lemmas.

LEMMA 2.6. $0 \rightarrow Q \xi H^{*} \rightarrow \hat{Q} \rightarrow Q$ is exact in all degrees.

Proof. Choose a Borel decomposition of $H^{*}$ with generators $\left\{x_{i}\right\}$. Then the elements $\left\{x_{i}\right\} \cup\left\{x_{i} x_{j} \neq 0\right\}$ give a basis of $Q_{2} H^{*}$. It is an easy coalgebra argument to show that $\hat{Q}=\operatorname{ker}\left\{\bar{\mu}^{*}: Q_{2} H^{*} \rightarrow \mathrm{Q} \otimes Q\right\}$ has a basis consisting of the elements $\left\{x_{i}^{2} \neq 0\right\}$ plus a subset of $\left\{x_{i}\right\}$. Q.E.D.

LEMMA 2.7. $\hat{Q}^{\text {even }} \rightarrow Q^{\text {even }}$ is surjective.

Proof. Pick $\bar{x} \in Q^{2 k}$. We want to show that $\bar{x}$ has a representation $x \in H^{*}$ such that $\bar{\mu}^{*} x=0$ in $Q \otimes Q$. Pick a basis $\mathscr{B}$ of $Q$. Then $\mathscr{B} \otimes \mathscr{B}$ is a basis of $Q \otimes Q$. Expand $\bar{\mu}^{*} x \in Q \otimes Q$ in terms of this basis. Given $u \in \mathscr{B}$ then $u \otimes u$ does not appear in $\bar{\mu}^{*} x$.

Given $u, v \in \mathscr{B}$ then

$(* *) \quad u \otimes v$ appears in $\bar{\mu}^{*} x$ if and only if $v \otimes u$ appears in $\bar{\mu}^{*} x$.

These restrictions follow from 2.3 and 2.4. We can use them to rewrite $x$ so that $\bar{\mu} x=0$ in $Q \otimes Q$. For if $u \otimes v$ (and hence $v \otimes u$ ) appears in $\bar{\mu}^{*} x$, replace $x$ by $x-u^{\prime} v^{\prime}$ where $u^{\prime}$ and $v^{\prime}$ are any representative in $H^{*}$ for $u$ and $v$. Q.E.D.

Lastly we want to show

LEMMA 2.8. $\mathrm{Sq}^{1} \hat{Q}^{\text {even }}=0$.

Proof. By 2.6 the map $\hat{Q}^{\text {odd }} \rightarrow Q^{\text {odd }}$ is injective. Lemma 2.8 is then a corollary of 2.5. Q.E.D. 
3. Relations in $\mathscr{A}(2)$. Our argument will require various relations in $\mathscr{A}(2)$. We will think of $\mathscr{A}(2)$ in terms of Milnor's description (see [12]). Milnor observed that $\mathscr{A}(2)$ is a Hopf algebra. Moreover, if $\mathscr{A}_{*}(2)$ is the dual Hopf algebra, then

$$
\mathscr{A}_{*}(2)=\mathbf{Z} / 2\left[\xi_{1}, \xi_{2}, \ldots\right], \quad\left|\xi_{k}\right|=2^{k}-1,
$$

and the coproduct on $\mathscr{A}_{*}(2)$ is determined by the rule

$$
\psi \xi_{k}=\sum_{i+j=k} \xi_{i}^{2 \prime} \otimes \xi_{j}
$$

If we dualize (3.1) and (3.2), then we have a complete description of $\mathscr{A}(2)$. Given an exponential sequence $R=\left(r_{1}, r_{2}, \ldots\right)$ let $\xi(R)=\xi_{1}^{r_{1}} \xi_{2}^{r_{2}} \cdots \xi_{k}^{r_{k}}$. Then $\{\xi(R)\}$ is a $\mathrm{Z} / 2$ basis of $\mathscr{A}_{*}(2)$. Dualizing we obtain a basis $\left\{\mathrm{Sq}^{R}\right\}$ of $\mathscr{A}(2)$. In particular, $\mathrm{Sq}^{(n, 0,0, \ldots)}=\mathrm{Sq}^{n}$, the usual Steenrod operation. The operations $\left\{\mathrm{Sq}^{n}\right\}$ obey a complicated multiplication rule which can be obtained by dualizing (3.2). All of the following relations can be established by using this rule of multiplication.

Let $\left.\Delta_{k}=0, \ldots, 0,1,0, \ldots\right)$ ( 1 is in the $k$ th position). Then $\mathrm{Sq}^{\Delta_{k}}$ is dual to $\xi_{k}$. The operations $\left\{\mathrm{Sq}^{\Delta_{s}}\right\}$ satisfy the relations

$$
\begin{array}{ll}
\mathrm{Sq}^{\Delta_{s}} \mathrm{Sq}^{\Delta_{t}}=\mathrm{Sq}^{\Delta_{t}} \mathrm{Sq}^{\Delta_{\triangleleft}}, & s, t \geqslant 1, \\
\left(\mathrm{Sq}^{\Delta_{s}}\right)^{2}=0, & s \geqslant 1 .
\end{array}
$$

In addition they satisfy the recursive relation

$$
\begin{aligned}
& \mathrm{Sq}^{\Delta_{1}}=\mathrm{Sq}^{1}, \\
& \mathrm{Sq}^{\Delta_{s+1}}=\mathrm{Sq}^{2^{s}} \mathrm{Sq}^{\Delta_{s}}+\mathrm{Sq}^{\Delta_{s}} \mathrm{Sq}^{2^{s}}, \quad s \geqslant 1 .
\end{aligned}
$$

For any $m>0$ and $s \geqslant 1$ we have the relation

$$
\mathrm{Sq}^{2 m+1}=\sum_{1 \leqslant t \leqslant s-1} \mathrm{Sq}^{2 m-2^{t}+2} \mathrm{Sq}^{\Delta_{t}}+\mathrm{Sq}^{\Delta_{s}} \mathrm{Sq}^{2 m-2^{s}+2}
$$

In particular, if we choose $s$ large, then the final term is trivial $\left(\mathrm{Sq}^{i}=0\right.$ for $\left.i<0\right)$ and, so we have

$$
\mathrm{Sq}^{2 m+1}=\sum_{t \geqslant 1} \mathrm{Sq}^{2 m-2^{t+2}} \mathrm{Sq}^{\Delta_{t}}
$$

Since $\mathrm{Sq}^{2^{s+1}-1} \mathrm{Sq}^{2^{s+1} k-2^{s}}=0$ we can modify (3.6) in the case $2 m=2^{s+1} k+2^{s}-2$ to obtain

$$
\begin{aligned}
\mathrm{Sq}^{2^{s+1} k+2^{s}-1}= & \sum_{1 \leqslant t \leqslant s} \mathrm{Sq}^{2^{s+1} k+2^{s}-2^{t} \mathrm{Sq}^{\Delta_{t}}} \\
& +\left(\mathrm{Sq}^{\Delta_{s+1}}+\mathrm{Sq}^{2^{s+1}-1}\right) \mathrm{Sq}^{2^{s+1} k-2^{s}} .
\end{aligned}
$$

Identities of the type (3.5) and (3.6) can be generalized. For any $R=\left(r_{1}, r_{2}, \ldots\right)$ and $s \geqslant 1$ there is the relation

$$
\mathrm{Sq}^{\Delta_{s}} \mathrm{Sq}^{R}=\mathrm{Sq}^{R} \mathrm{Sq}^{\Delta_{s}}+\sum_{i \geqslant 0} \mathrm{Sq}^{\Delta_{s+i}} \mathrm{Sq}^{R-2^{s} \Delta_{i}}
$$


(Here $2^{s} \Delta_{i}=\left(0,0, \ldots, 0,2^{s}, 0, \ldots\right)$ with $2^{s}$ occurring in the $i$ th position, and $\mathrm{Sq}^{R-2^{s} \Delta_{i}}$ $=0$ if $r_{i}<2^{s}$.) We can deduce from (3.8) that, for each $R$ and $s \geqslant 1$, there exists a relation of the form

$$
\mathrm{Sq}^{\Delta_{s}} \mathrm{Sq}^{R}=\mathrm{Sq}^{R} \mathrm{Sq}^{\Delta_{s}}+\sum \mathrm{Sq}^{R_{j}} \mathrm{Sq}^{\Delta_{t_{j}}}
$$

where $t_{j}>s$. There are some cruder relations which we will also have need of. Let $\left(\theta_{1}, \ldots, \theta_{k}\right)$ be the two sided ideal of $\mathscr{A}(2)$ generated by $\theta_{1}, \ldots, \theta_{k}$.

$$
\begin{aligned}
& \mathrm{Sq}^{2^{s}} \mathrm{Sq}^{2^{s} k} \equiv 0 \bmod \sum_{i=0}^{s-1}\left(\mathrm{Sq}^{2^{i}}\right)^{2} \text { for } k \text { odd }, \\
& \mathrm{Sq}^{2^{s}} \mathrm{Sq}^{2^{s+1}} \equiv \mathrm{Sq}^{2^{s+1} k+2^{s}} \bmod \sum_{i=0}^{s-1}\left(\mathrm{Sq}^{2^{i}}\right)^{2}
\end{aligned}
$$

First of all, these relations hold $\bmod \left(\mathrm{Sq}^{1}, \mathrm{Sq}^{2}, \ldots, \mathrm{Sq}^{2^{s-1}}\right) . \mathscr{A}(2) /\left(\mathrm{Sq}^{1}, \mathrm{Sq}^{2}, \ldots, \mathrm{Sq}^{2^{s-1}}\right)$ $\cong \mathscr{A}(2)$ as algebras, where $\mathrm{Sq}^{2^{s+i}} \in \mathscr{A}(2) /\left(\mathrm{Sq}^{1}, \mathrm{Sq}^{2}, \ldots, \mathrm{Sq}^{2^{s-1}}\right)$ corresponds to $\mathrm{Sq}^{2^{i}}$ $\in \mathscr{A}(2)$. And in $\mathscr{A}(2)$ we have the relation $\mathrm{Sq}^{1} \mathrm{Sq}^{k}=0$ for $k$ odd and $\mathrm{Sq}^{1} \mathrm{Sq}^{2 k}=$ $\mathrm{Sq}^{2 k+1}$. Secondly, any element $\theta \in\left(\mathrm{Sq}^{1}, \mathrm{Sq}^{2}, \ldots, \mathrm{Sq}^{2^{s-1}}\right)$ having degree $\equiv 0 \bmod 2^{s}$ must have at least one of $\left\{\mathrm{Sq}^{1}, \mathrm{Sq}^{2}, \ldots, \mathrm{Sq}^{2^{s-1}}\right\}$ appearing twice when we expand $\theta$ as a polynomial in the indecomposable operations $\left\{\mathrm{Sq}^{2^{i}}\right\}_{i \geqslant 0}$.

4. Secondary operations. The main tool of the next two sections will be secondary operations. For the general theory of such operations see [ 7 or 17]. For the specific predecessors of the mod 2 arguments which we will employ in this paper see $[4,5$ and 8]. In this section we summarize various facts which will be required in applying our secondary operations. We will also describe some of the arguments which we will be employing.

The main structure theorem for secondary operations is the following

Theorem 4.1. Let $(X, \mu)$ be an H-space. Pick $x \in H^{2 n}$ and $B \subset H^{*}$, a sub-Hopf algebra invariant under $\mathscr{A}(2)$, where $\bar{\mu}^{*} x \in B \otimes B$. Suppose that in degree $2 n-|\theta|+1$ we have the relation

$$
\mathrm{Sq}^{2 n+1} \boldsymbol{\theta}=\sum_{i=1}^{l} a_{i} b_{i}
$$

where $x=\theta y$ and $b_{i} y \in \bar{B} \cdot \bar{B}$ for $1 \leqslant i \leqslant l$. Then there is a secondary operation $\phi$ defined on $y$ which satisfies

$$
\bar{\mu}^{*} \phi(y)=x \otimes x+\sum_{i} \operatorname{Im} a_{i}
$$

in $H^{*} / / B \otimes H^{*} / / B$.

We will employ 4.1 as follows. Let $x, B$ and $\mathrm{Sq}^{2 n+1} \theta=\sum a_{i} b_{i}$ be as in Theorem 4.1. Suppose we pick $\alpha \in P_{2 n}$, where $\langle\alpha, x\rangle \neq 0,\langle\alpha, B\rangle=0$. Since $\alpha^{2}=0$ we must have

$$
\left\langle\alpha \otimes \alpha, \operatorname{Im} a_{i}\right\rangle \neq 0 \quad \text { for some } i
$$


Otherwise $\left\langle\alpha^{2}, \phi(y)\right\rangle=\langle\alpha \otimes \alpha, \bar{\mu} \phi(y)\rangle=\langle\alpha \otimes \alpha, x \otimes x\rangle \neq 0$. The fact that $(\alpha \otimes \alpha) a_{i} \neq 0$ for some $i$ forces operations from $\mathscr{A}(2)$ to act nontrivially on $\alpha$ (use the Cartan formula). Dualizing, we have $\mathscr{A}(2)$ acting nontrivially on $Q$. This is the manner in which we will study the Steenrod module structure of $Q$.

Any application of Theorem 4.1 involves verifying the hypothesis of the theorem. Supppose we have $\bar{x}=\theta \bar{y}$ and a relation $\mathrm{Sq}^{2 n+1}=\sum a_{i} b_{i}$. If $\theta \neq 1$ (and thus $\bar{y} \neq \bar{x}$ ) it is generally very difficult to pick representatives $x, y \in H^{*}$ and an invariant Hopf algebra $B \subset H^{*}$ such that $x \notin B, \bar{\mu}^{*} x \in B=B$ and $b_{i} y \in B \cdot B$. So let us restrict our attention to the case $\theta=1, \bar{y}=\bar{x}$. Assume we have $\bar{x} \in Q^{2 n}$ and a fixed relation $\mathrm{Sq}^{2 n+1}=\sum_{i} a_{i} b_{i}$.

(A) The sub-Hopf algebra $B$. We can always choose a representative $x \in H^{2 n}$ and a sub-Hopf algebra $B$ such that $x \notin B$ and $\bar{\mu}^{*} x \in B \otimes B$. Let $B(q)=$ the sub-Hopf algebra of $H^{*}$ generated, over $\mathscr{A}(2)$, by $\sum_{i \leqslant q} H^{i}$. The Hopf algebras $\{B(q)\}$ induce a filtration $\left\{F_{q} Q\right\}$ of $Q$. Suppose $\bar{x} \in F_{q+1} Q, x \notin F_{q} Q$. Pick a representative $x \in$ $B(q+1)$ and let $B=B(q)$. By construction $\bar{\mu}^{*} x \in B \otimes B$.

(B) The relation $b_{i} x \in \bar{B} \cdot \bar{B}$. Showing that $b_{i} x \in \bar{B} \cdot \bar{B}$ requires more analysis. The following lemmas play a pivotal role.

Lemma 4.2. Let $A \subset A_{0}$ be a commutative, associative Hopf algebra. Given $z \in A_{0}$, where $\bar{\mu}^{*} z \in A \otimes A$, then $z \in A$ if and only if $z=0$ in $A_{0} / / A$.

LEMMA 4.3. For any commutative, associative Hopf algebra $A$

(i) $P^{2 k+1} A \cap D^{2} A=0$,

(ii) $P^{4 k+2} A \cap D^{3} A=0$.

For proofs of these lemmas see $[3,3.1,3.2]$. We can use these lemmas to prove, that in many instances, $b_{i} x \in \bar{B} \cdot \bar{B}$. Suppose $x \in H^{*}$ is a representative for $\bar{x} \in \hat{Q}$ $\subset Q_{2} H^{*}$. Then $b_{i} \bar{x} \in \hat{Q}$ as well.

Lemma 4.4. If $\bar{x} \in \hat{Q}, \operatorname{deg} b_{i} \bar{x} \neq \equiv \bmod 4$ and $b_{i} \bar{x}=0$ in $\hat{Q}$, then $b_{i} x \in \bar{B} \cdot \bar{B}$.

Proof. First of all, $b_{i} x \in B$. For, by 4.2 , we want to show $b_{i} x=0$ in $H^{*} / / B$. We have $b_{i} x \in P\left(H^{*} / / B\right)$. Also, $b_{i} \bar{x}=0$ in $\hat{Q}$ implies $b_{i} x \in D^{3}\left(H^{*} / / B\right)$. By 4.3 $b_{i} x x=0$.

Secondly $b_{i} x \in \bar{B} \cdot \bar{B}$. Let $B^{\prime}$ be the sub-Hopf algebra of $B$ generated by elements of degree $<\left|b_{i} x\right|$. If $b_{i} x$ is indecomposable in $B$, then $b_{i} x \neq 0$ in $B / / B^{\prime} \subset H^{*} / / B^{\prime}$. But an analogue of our previous argument shows that $b_{i} x \in P\left(H^{*} / / B^{\prime}\right) \cap$ $D^{3}\left(H^{*} / / B^{\prime}\right)=0$. Q.E.D.

Lemma 4.4 is particularly useful because of the following extension of Lemma 2.8 .

Lemma 4.5. If $\bar{x} \in \hat{Q}^{\text {even }}$, then $\mathrm{Sq}^{\Delta} \bar{x}=0$ in $\hat{Q}$ for $i \geqslant 1$.

(C) The primitive $\alpha$. Although it is not directly mentioned in Theorem 4.1 we will also need to pick $\alpha \in P$, where $\langle\alpha, x\rangle \neq 0,\langle\alpha, B\rangle=0$. Let $\left(H^{*} / / B\right)^{*} \subset H_{*}$ be the dual Hopf algebra of $H^{*} / / B$. Then $P\left(H^{*} / / B\right)^{*} \subset P$. Since $\bar{x} \neq 0$ in $Q\left(H^{*} / / B\right)$ we can pick $\alpha \in P\left(H^{*} / / B\right)^{*}$ where $\langle\alpha, x\rangle \neq 0$. By construction $\langle\alpha, B\rangle=0$. 
5. Proof of Theorem 1.2, part I. In this section we obtain a great deal of information about $\hat{Q}^{\text {even }}$. In particular we prove that $\hat{Q}^{\text {even }}$ is generated over $\mathscr{A}(2)$ by $\sum_{s \geqslant 2} \hat{Q}^{2^{s}-2}$. The results and proofs of this section are modelled on those of Lin in [8]. In this section we prove the results of [8] without using the hypothesis that $H_{*}$ is associative. We will prove

THEOREM 5.1. If $k \geqslant 1$ and $s \geqslant 1$, then $\hat{Q}^{2^{s+1} k+2^{s}-2} \subset \operatorname{Im~Sq}{ }^{2^{s}}+\operatorname{Im~Sq}{ }^{2^{s} k}$.

It is a simple consequence of 5.1 that

COROLLARY 5.2. $\hat{Q}^{\text {even }}$ is generated over $\mathscr{A}(2)$ by $\sum_{s \geqslant 2} \hat{Q}^{2^{s}-2}$.

Another, less obvious, consequence of 5.1 is

Corollary 5.3. If $k \geqslant 1$ and $s \geqslant 1$, then $\mathrm{Sq}^{2^{s}} \hat{Q}^{2^{s+1} k+2^{s}-2}=0$.

Corollary 5.3 is equivalent to asserting that $\hat{Q}^{2^{s+1} k+2^{s+1}-2} \cap \operatorname{Im~Sq}{ }^{s}=0$ for $k$, $s \geqslant 1$. One can easily extend Corollary 5.3 to assert

Corollary 5.4. If $k \geqslant 1$ and $s \geqslant 1$, then $\hat{Q}^{2^{s+1} k+2^{s+1}-1} \cap \operatorname{Im~Sq}{ }^{2 t}=0$ unless $2 t \equiv 0 \bmod 2^{s+1}$.

We will prove Theorem 5.1 and Corollaries 5.3 and 5.4 by an inductive argument. Corollaries 5.3 and 5.4 will be required for the inductive step in the proof of Theorem 5.1. So, we will prove 5.1, 5.3 and 5.4 simultaneously. Our proofs will be by induction on $s$.

(A) The initial case. For $s=1$, Theorem 5.1 becomes $\hat{Q}^{4 k} \subset \operatorname{Im~Sq}{ }^{2}+\operatorname{Im~Sq}^{2 k}$. Actually, a stronger result holds, namely $\hat{Q}^{4 k} \subset \operatorname{Im~Sq}{ }^{2 k}$. The exact sequence (1.1) plus the triviality of $Q^{4 k}$ for $k \geqslant 1$ gives an isomorphism $Q^{4 k}\left(\xi H^{*}\right) \cong \hat{Q}^{4 k}$, and $Q^{4 k}\left(\xi H^{*}\right)=\mathrm{Sq}^{2 k} \hat{Q}^{2 k}$.

For Corollary 5.3 we must show $\mathrm{Sq}^{2} \hat{Q}^{4 k}=0$. We have the identities

$$
\mathrm{Sq}^{2} \hat{Q}^{4 k}=\mathrm{Sq}^{2} \mathrm{Sq}^{2 k} \hat{Q}^{2 k}=\left(\mathrm{Sq}^{2 k+2}+\mathrm{Sq}^{2 k+1} \mathrm{Sq}^{1}\right) \hat{Q}^{2 k}=0 .
$$

In the last identity $\mathrm{Sq}^{2 k+2} \hat{Q}^{2 k}=0$ since $\mathscr{A}(2)$ acts unstably while $\mathrm{Sq}^{2 k+1} \mathrm{Sq}^{1} \hat{Q}^{2 k}=0$ by 2.8 .

Finally, Corollary 5.4 follows trivially from Corollary 5.3.

(B) The induction hypothesis. Fix $s \geqslant 2$. Assume that 5.15 .3 and 5.4 hold for $s^{\prime}<s$. We will first prove Theorem 5.1 for the case $s$ and then the corollaries. Fix $k \geqslant 1$ and assume that Theorem 5.1 holds for $\hat{Q}^{2^{s+1} k^{\prime}+2^{s}-2}$ when $k^{\prime}>k$. We will divide our proof of Theorem 5.1 into three cases. Pick $\bar{x} \in \hat{Q}^{2^{s+1} k+2^{s}-2}$.

(i) $\bar{x} \in \hat{Q} \xi H^{*} \subset \hat{Q}$,

(ii) $k>1$ and $\bar{x} \neq 0$ in $Q$.

(iii) $k=1$ and $\bar{x} \neq 0$ in $Q$.

(C) The case $\bar{x} \in Q \xi H^{*}$. To deal with this case we must pass from $\hat{Q}$ to a subquotient module $\bar{Q}$ of $Q_{2} H^{*}$. Let

$$
\begin{aligned}
& \hat{\hat{Q}}^{\text {odd }}=\left\{x \in Q_{2}^{\text {odd }} H^{*} \mid \bar{\mu}^{*} x \in Q^{\text {odd }} \otimes Q^{\text {even }}\right\}, \\
& \hat{\hat{Q}}^{\text {even }}=\left\{x \in Q_{2}^{\text {even }} H^{*} \mid \bar{\mu}^{*} x \in Q^{\text {even }} \otimes Q^{\text {even }}\right\} .
\end{aligned}
$$


Obviously $\hat{Q} \subset \hat{\hat{Q}}$. Let $\bar{Q}$ be the quotient algebra

$$
\bar{Q}=\hat{\hat{Q}} / \hat{\hat{Q}}^{\text {even }} \cdot \hat{\hat{Q}}^{\text {even }} \text {. }
$$

By naturality, plus the fact that $\mathrm{Sq}^{1} Q^{\text {even }}=0$, the action of $\mathscr{A}(2)$ on $Q_{2} H^{*}$ induces an action on $\hat{Q}$. As in $2.8 \mathrm{Sq}^{1} \hat{Q}^{\text {even }}=0$. So there is an induced action of $\mathscr{A}(2)$ on $\bar{Q}$. We have a short exact sequence

$$
0 \rightarrow Q \xi H^{*} \rightarrow \bar{Q} \rightarrow Q \rightarrow 0
$$

in degrees $\not \equiv 0 \bmod 4$. (In degrees $4 k$ the map $Q \xi H^{*} \rightarrow \bar{Q}$ is trivial.) The proof of exactness is an extension of the proof given in 2.6 and 2.7 for the exactness of (1.1). By comparing (5.5) with (1.1) it follows that the natural map $\hat{Q}^{\text {even }} \rightarrow \hat{\hat{Q}}^{\text {even }} \rightarrow \bar{Q}^{\text {even }}$ induces an isomorphsm.

$$
\hat{Q}^{\text {even }} \cong \bar{Q}^{\text {even }} \quad \text { as } \mathscr{A}(2) \text { modules in degrees } \not \equiv 0 \bmod 4 .
$$

We now deal with the case $\bar{x} \in Q \xi H^{*}$. We will prove that $Q \xi H^{*} \subset \hat{Q}$ satisfies the following strengthened version of 5.1 .

LEMma 5.7. $Q^{2^{s+1} k+2^{s-2}}\left(\xi H^{*}\right) \subset \operatorname{Im~Sq}{ }^{2^{s} k}$.

Proof. By (5.6) we can replace $\hat{Q}$ by $\bar{Q}$ and consider $Q \xi H^{*} \subset \bar{Q}$. Given $\bar{x} \in$ $Q \xi H^{*} \subset Q$, by (5.5) we have $\bar{x}=\bar{y}^{2}$ for $\bar{y} \in \bar{Q}^{2^{s} k+2^{s-1}-1}$. Then

$$
\begin{aligned}
\bar{y}^{2} & =\mathrm{Sq}^{2^{s} k+2^{s-1}-1}(\bar{y}) \\
& =\sum_{1 \leqslant t \leqslant s-1} \mathrm{Sq}^{2^{s} k+2^{s-1}-2^{t}} \mathrm{Sq}^{\Delta t}(\bar{y})+\left(\mathrm{Sq}^{\Delta_{s}}+\mathrm{Sq}^{2^{s}-1}\right) \mathrm{Sq}^{2^{s}(k-1)+2^{s-1}}(\bar{y}) \\
& =\mathrm{Sq}^{2^{s} k} \mathrm{Sq}^{\Delta_{s-1}}(\bar{y}) .
\end{aligned}
$$

The second identity is relation (3.7). For the last identity we use Corollary 5.4 (íor $\left.s^{\prime}<s\right)$ to deduce

$$
\bar{Q}^{2^{s+1} k+2^{s}-2} \cap \operatorname{Im~Sq}^{2^{s} k+2^{s} 1^{1}-2^{\prime}}=0 \quad(1 \leqslant t \leqslant s-2)
$$

and 5.4 plus the identity $\mathrm{Sq}^{\Delta_{s}}+\mathrm{Sq}^{2^{s}-1}=\sum_{1 \leqslant i \leqslant s-1} \mathrm{Sq}^{2^{s}-2^{\prime}} \mathrm{Sq}^{\Delta_{i}}$ to deduce that

$$
\bar{Q}^{2^{s+1} k+2^{s}-2} \cap \operatorname{Im}\left(\mathrm{Sq}^{\Delta_{s}}+\mathrm{Sq}^{2^{s}-1}\right)=0 \text {. }
$$

(D) The case $k \geqslant 1$ and $\bar{x} \neq 0$ in $Q$. Suppose $\bar{x} \in F_{q+1} Q, \bar{x} \notin F_{q} Q$. We can assume, by induction, that Theorem 5.1 holds for $F_{q} Q$ in the case of our fixed $s$. As in $\$ 4$ pick $\in H^{2^{s+1} k+2^{s}-2}, B=B(q) \subset H^{*}$ and $\alpha \in P_{2^{s+1} k+2^{s}-2}$, where $x \notin B$, $\bar{\mu}^{*}(x) \in B \otimes B,\langle\alpha, x\rangle \neq 0$ and $\langle\alpha, B\rangle=0$. We want to show that $\alpha \mathrm{Sq}^{2} \neq 0$ or $\alpha \mathrm{Sq}^{2^{s} k} \neq 0$. We will suppose the opposite and force $\alpha^{2} \neq 0$, a contradiction to Lemma 2.4. To show that $\alpha^{2} \neq 0$ we will use the secondary operation $\phi$ associated with the relation




The operation is defined on $x$. Namely

LEMMA 5.8. (i) $\mathrm{Sq}^{\Delta_{t}}(x) \in \bar{B} \cdot \bar{B}$.

(ii) $\mathrm{Sq}^{2^{s+1} k-2^{s}}(x) \in \bar{B} \cdot \bar{b}$.

Proof. By 4.4 we need only show

$$
\operatorname{Sq}^{\Delta_{t}}(\bar{x})=\operatorname{Sq}^{2^{s+1} k-2^{s}}(\bar{x})=0
$$

in $\hat{Q}$. For the identity $\operatorname{Sq}^{\Delta_{t}}(\bar{x})=0$ use 4.5 . For the second identity we need only show

$$
\mathrm{Sq}^{2^{s+1} k-2^{s}}(\bar{x}) \in \sum_{i=0}^{s-1} \operatorname{Im}\left(\mathrm{Sq}^{2^{i}}\right)^{2}
$$

(Here $\left(\mathrm{Sq}^{2^{i}}\right)$ denotes the ideal generated by $\mathrm{Sq}^{2^{i}}$.) For it follows from Corollary 5.4 (for $s^{\prime}<s$ ) plus 2.8 that $\hat{Q}^{2^{s+2} k-2} \cap \operatorname{Im}\left(\mathrm{Sq}^{2^{i}}\right)^{2}=0$ when $1 \leqslant i \leqslant s$. So let us prove (5.8.1). By (3.11) we have

$$
\mathrm{Sq}^{2^{s+1} k-2^{s}} \equiv \mathrm{Sq}^{2^{s}} \mathrm{Sq}^{2^{s+1}(k-1)} \bmod \sum_{i=0}^{s-1}\left(\mathrm{Sq}^{2^{i}}\right)^{2}
$$

Also, we have

$$
\mathrm{Sq}^{2^{s+1}(k-1)}(\bar{x}) \in \hat{Q}^{2^{s+1}(2 k-1)+2^{s}-2} \subset \operatorname{Im~Sq} 2^{2^{s}(2 k-1)}+\operatorname{Im~Sq}{ }^{2^{s}}
$$

(Here we use the assumption that 5.1 is true for $k^{\prime}>k$ is as well as the assumption that $k>1)$. Writing $\operatorname{Sq}^{2^{s+1}(k-1)}(\bar{x})=\operatorname{Sq}^{2^{s}(2 k-1)}(\bar{y})+\operatorname{Sq}^{2^{s}}(\bar{z})$ we have

$$
\begin{aligned}
\mathrm{Sq}^{2^{s}} \mathrm{Sq}^{2^{s+1}(k-1)}(\bar{x}) & =\mathrm{Sq}^{2^{s}} \mathrm{Sq}^{2^{s}(2 k-1)}(\bar{y})+\mathrm{Sq}^{2^{s}} \mathrm{Sq}^{2^{s}}(\bar{z}) \\
& \equiv 0 \bmod \sum_{i=0}^{s-1} \operatorname{Im}\left(\mathrm{Sq}^{2^{i}}\right)^{2} .
\end{aligned}
$$

For the last equality we use (3.10). By combining (5.8.2) and (5.8.3) we deduce (5.8.1). Q.E.D.

We will prove that $\alpha^{2} \neq 0$ by showing $\left\langle\alpha^{2}, \phi(x)\right\rangle \neq 0$. By 4.1 we have

$$
\bar{u}^{*} \phi(x)=x \otimes x+\sum_{1 \leqslant t \leqslant s} \operatorname{Im} \mathrm{Sq}^{2^{s+1} k+2^{s}-2^{t}}+\operatorname{Im}\left(\mathrm{Sq}^{\Delta_{s+1}}+\mathrm{Sq}^{2^{s+1}-1}\right) .
$$

We want to show that $\alpha \otimes \alpha$ annihilates all the terms in the right-hand side of (5.9) except $x \otimes x$. For then

$$
\begin{aligned}
\left\langle\alpha^{2}, \phi(x)\right\rangle & =\left\langle\bar{\mu}_{*}(\alpha \otimes \alpha), \phi(x)\right\rangle=\left\langle\alpha \otimes \alpha, \bar{\mu}^{*} \phi(x)\right\rangle \\
& =\langle\alpha \otimes \alpha, x \otimes x\rangle \neq 0 .
\end{aligned}
$$

By Corollary 5.4 (for $s^{\prime}<s$ ) plus our assumption that $\alpha \mathrm{Sq}^{2^{s}}=\alpha \mathrm{Sq}^{2^{s+1}} k=0$ we have

LEMMA 5.10. $\alpha \mathrm{Sq}^{2 i}=0$ unless $2 i \equiv 0 \bmod 2^{s+1}$.

LEMMA 5.11. $(\alpha \otimes \alpha)\left(\mathrm{Sq}^{\Delta_{s+1}}+\mathrm{Sq}^{2^{s+1}-1}\right)=0$. 
Proof. By (3.2)

$$
(\alpha \otimes \alpha)\left(\mathrm{Sq}^{\Delta_{s+1}}+\mathrm{Sq}^{2^{s+1}-1}\right)=\sum_{1 \leqslant i \leqslant s}(\alpha \otimes \alpha) \mathrm{Sq}^{2^{s+1}-2^{i}} \mathrm{Sq}^{\Delta_{\prime}}
$$

On the other hand, for $1 \leqslant i \leqslant s$

$$
(\alpha \otimes \alpha) \mathrm{Sq}^{2^{s+1}-2^{i}} \mathrm{Sq}^{\Delta_{i}}=\sum_{2 u+2 v=2^{s+1}-2^{i}}\left(\alpha \mathrm{Sq}^{2 u} \otimes \alpha \mathrm{Sq}^{2 v}\right) \mathrm{Sq}^{\Delta_{i}}=0 .
$$

The first identity follows from the Cartan formula plus 2.5. The second identity follows from 5.10. Q.E.D.

LEMMA 5.12. $(\alpha \otimes \alpha) \mathrm{Sq}^{2^{s+1} k+2^{s}-2^{t}}=0$ for $1 \leqslant t \leqslant s$.

Proof. Expand

$$
(\alpha \otimes \alpha) \mathrm{Sq}^{2^{s+1} k+2^{s}-2^{\prime}}=\sum_{i+j=2^{s+1} k+2^{s}-2^{t}} \alpha \mathrm{Sq}^{i} \otimes \alpha \mathrm{Sq}^{j} .
$$

(i) $i$ and $j$ odd. Since $i+j \geqslant 2^{s+1} k$ and $\alpha \mathrm{Sq}^{2^{s} k}=0$ it follows that either $i$ or $j>2^{s} k$. Suppose $i>2^{s} k$. Then $i=2^{s} k+q, 0<q<2^{s}-1$. (To derive the upper bound on $q$ we use the fact that $\mathscr{A}(2)$ acts unstably on $H^{*}$.) We will show that $\alpha \mathrm{Sq}^{i}=0$ for each value of $q$.

(a) $q=2^{r}-1$. We have the relation

$$
\alpha \mathrm{Sq}^{2^{s} k+2^{r}-1}=\sum_{1 \leqslant t \leqslant r} \alpha \mathrm{Sq}^{2^{s} k+2^{r}-2^{t}} \mathrm{Sq}^{\Delta_{t}}+\alpha\left(\mathrm{Sq}^{\Delta_{r+1}}+\mathrm{Sq}^{2^{r+1}-1}\right) \mathrm{Sq}^{2^{s} k-2^{r}}
$$

By 5.10, plus the assumption that $\alpha \mathrm{Sq}^{2 \cdot k}=0$, it follows that every term in the right-hand side is trivial. In particular, we must expand

$$
\mathrm{Sq}^{\Delta_{r+1}}+\mathrm{Sq}^{2^{r+1}-1}=\sum_{1 \leqslant p \leqslant r} \mathrm{Sq}^{2^{r+1}-2^{p}} \mathrm{Sq}^{\Delta_{p}}
$$

to make use of 5.10 .

(b) $q \neq 2^{r}-1$. By an argument similar to the above, $\alpha \mathrm{Sq}^{2^{s} k+q}=0$. We use relation (3.3) plus 5.10.

(ii) $i$ and $j$ even. By 5.10 we can reduce to the case $i \equiv j \equiv 0 \bmod 2^{s+1}$. Since $i+j=2^{s+1} k+2^{s}-2^{t}$ we must have $s=t$. So $i=2^{s+1} k^{\prime}$ and $j=2^{s+1} k^{\prime \prime}$, where $k^{\prime}+k^{\prime \prime}=k$. Since $\mathscr{A}(2)$ acts unstably we can eliminate all cases except $k=2 l$, $k^{\prime}=k^{\prime \prime}=l$. This case can also be eliminated since we are assuming that $\alpha \mathrm{Sq}^{2^{\circ} k}=0$. Q.E.D.

(E) The case $k=1$ and $\bar{x} \neq 0$ in $Q$. Suppose $\bar{x} \in F_{q+1} Q, \bar{x} \notin F_{q} Q$. We can assume, by induction, that Theorem 5.1 holds for the elements of $F_{q} Q$ in the case $s$. As in $\S 4$ pick a representative $x \in H^{2^{s+1}+2^{s}-2}, B=B(q) \subset H^{*}$, and $\alpha \in P_{2^{s+1}+2^{s}-2}$, where $x \notin B, \bar{\mu}^{*}(x) \in B \otimes B,\langle\alpha, x\rangle \neq 0$ and $\langle\alpha, B\rangle=0$. We want to show that $\alpha \mathrm{Sq}^{2^{3}} \neq$ 0 . It suffices to show that $\alpha \mathrm{Sq}^{2 k} \neq 0$ for some $k>0$. For, then, $\alpha \mathrm{Sq}^{2} \neq 0$ for some $i \geqslant 1$. We can eliminate $i>s$ since $\mathscr{A}(2)$ acts unstably on $H^{*}$. We can eliminate $i<s$ by Corollary 5.4 (for $s^{\prime}<s$ ). 
Our proof will be any contradiction. We will assume that $\alpha \mathrm{Sq}^{2 k}=0$ for $k>0$ and show that $\alpha^{2} \neq 0$, contradicting Lemma 2.4. First of all, we have

LEMmA 5.13. If $\operatorname{deg} \mathrm{Sq}^{R}=l$ and $\alpha \mathrm{Sq}^{R} \neq 0$, then $l=2^{i}-1$ and $\alpha \mathrm{Sq}^{R}=\alpha \mathrm{Sq}^{2^{i}-1}$.

Proof. First of all, $k$ is odd. For we can assume $\mathrm{Sq}^{R}=\mathrm{Sq}^{2^{i_{1}}} \mathrm{Sq}^{2^{i_{2}}} \cdots \mathrm{Sq}^{2^{i_{r}}}$. Since $\alpha \mathrm{Sq}^{2 k}=0$ for $k>0$ we have $i_{1}=0$. But then, by $2.5, i_{2}, \ldots, i_{r}$ are even. Second, we can write $\mathrm{Sq}^{R}=\mathrm{Sq}^{\Delta_{i}} \mathrm{Sq}^{R^{\prime}}$ for some $i \geqslant 1$ and $R^{\prime}$. By iterating relation (3.5) we can reduce $\mathrm{Sq}^{R}$ to the case

$$
\mathrm{Sq}^{R}=\mathrm{Sq}^{R^{\prime}} \mathrm{Sq}^{\Delta_{i}}
$$

for some $i \geqslant 1$ and $R^{\prime}$. If $\alpha \mathrm{Sq}^{R^{\prime}} \mathrm{Sq}^{\Delta_{i}} \neq 0$ then, by 2.5 plus the assumption that $\alpha \mathrm{Sq}^{2 k}=0$, we can force $\mathrm{Sq}^{R^{\prime}}=1$. Finally, using the relation $\mathrm{Sq}^{\Delta_{i}}=\mathrm{Sq}^{2^{i}-1}+$ $\sum_{j<i} \mathrm{Sq}^{2^{i}-2^{j}} \mathrm{Sq}^{\Delta_{j}}$ we have $\alpha \mathrm{Sq}^{\Delta_{i}}=\alpha \mathrm{Sq}^{2^{i}-1}$. Q.E.D.

On the other hand, we must have

LEMMA 5.14. $\alpha \mathrm{Sq}^{k} \neq 0$ for some $k \geqslant 1$.

Proof. We use the secondary operation associated with the relation



As in 5.8(i) we have $\mathrm{Sq}^{{ }_{t}}(x) \in \bar{B} \cdot \bar{B}$. So the secondary operation associated to (*) is defined on $x$ and satisfies

$$
\bar{\mu}^{*} \phi(x)=x \otimes x+\sum_{t \geqslant 1} \operatorname{Im~Sq} 2^{2^{s+1}+2^{s}-2^{t}}
$$

in $H^{*} / / B \otimes H^{*} / / B$. If $\left\langle\alpha \otimes \alpha, \operatorname{Im~Sq}{ }^{2^{s+1}-2^{s}-2^{t}}\right\rangle=0$ for $t \geqslant 1$, then $\left\langle\alpha^{2}, \phi(x)\right\rangle=$ $\langle\alpha \otimes \alpha, \bar{\mu} \phi(x)\rangle=\langle\alpha \otimes \alpha, x \otimes x\rangle \neq 0$. But $\alpha^{2} \neq 0$ contradicts Lemma 2.4. We

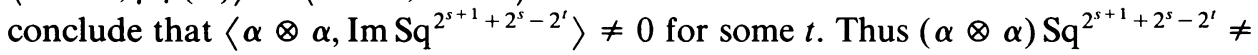
0 and, by the Cartan formula, $\alpha \mathrm{Sq}^{k} \neq 0$ for $k>0$. Q.E.D.

We now repeat the arguments used to prove Lemma 5.14 with enough significant modifications to deduce not just that $\alpha \mathrm{Sq}^{k} \neq 0$ but that $\alpha \mathrm{Sq}^{2 k} \neq 0$. Replace the relation (*) used in 5.14 with the relation

$$
\mathrm{Sq}^{2^{s+1}+2^{s}-1} \mathrm{Sq}^{1}=\sum_{t \geqslant 1}\left(\mathrm{Sq}^{2^{s+1}+2^{s}-2^{t}} \mathrm{Sq}^{1}\right) \mathrm{Sq}^{\Delta_{t}}
$$

The use of this relation demands a more subtle choice of the representative $x \in H^{2^{s+1}+2^{s}-2}$ and the sub-Hopf algebra $B \subset H^{*}$. Suppose

$$
\left\langle\alpha, F_{q} Q\right\rangle=0, \quad\left\langle\alpha, F_{q+1} Q\right\rangle \neq 0 .
$$

Pick $\bar{x} \in F_{q+1} Q$, where $\langle\alpha, \bar{x}\rangle \neq 0$. Next, we choose $\bar{y} \in Q^{2^{2+1}+2^{s}-3}$, where $\mathrm{Sq}^{1} \bar{y}=\bar{x}$, $\bar{y} \in F_{q+1} Q$ and $\bar{y} \notin F_{q} Q$. By 5.13 we can write $\bar{x}=\mathrm{Sq}^{2^{i}-1} \bar{z}$, where $|\bar{z}|=q+1$. Let $\bar{y}=\mathrm{Sq}^{2^{i}-2} \bar{z}$. The first two properties of $\bar{y}$ are immediate. The third property is then forced by the fact that $\bar{x} \notin F_{q} Q$. Let $B=$ the sub-Hopf algebra, invariant under $\mathscr{A}(2)$, generated by $B(q)$ and $\xi H^{*}$. (Here $B(q)$ is defined as in $\S 4$.) We have $\langle\alpha, B\rangle=0$. We can choose representatives $y$ and $x=\mathrm{Sq}^{1} y$ for $\bar{y}$ and $\bar{x}=\mathrm{Sq}^{1} \bar{y}$ so that $x \notin B$ and $\bar{\mu}^{*} y \in B \otimes B$. 
The main subtlety in our argument is showing that the operation $\phi$ associated to (5.15) is defined on $y$. This is the one point in the paper where the suggested approach from $\S 4$ for showing $b_{i}(y) \in \bar{B} \cdot \bar{B}$ does not apply. First of all we have

Lemma 5.16. $\mathrm{Sq}^{\Delta_{t}}(y) \in \bar{B}$ for $t \geqslant 1$.

Proof. Since $\bar{\mu}^{*}(y) \in B \otimes B$ it follows that $\bar{\mu}^{*} \operatorname{Sq}^{\Lambda_{t}}(y) \in B \otimes B$. It follows from 4.2 that we need only show $\operatorname{Sq}^{\Delta_{t}}(y)=0$ in $H^{*} / / B$. We have $\operatorname{Sq}^{\Delta}(y) \in$ $P^{4 i}\left(H^{*} / / B\right)$. Since $\xi H^{*} \subset B, H^{*} / / B$ is an exterior algebra and, by 4.20 of [13], the map $P\left(H^{*} / / B\right) \rightarrow Q\left(H^{*} / / B\right)$ is injective. By $2.1 Q^{4 i}\left(H^{*} / / B\right)=0$ for all $i$. Thus $P^{4 i}\left(H^{*} / / B\right)=0$ for all $i$. Q.E.D.

Because of 5.16 we can prove $\operatorname{Sq}^{{ }^{t}}(y) \in \bar{B} \cdot \bar{B}$ by showing that $Q^{4 i}(B)=0$ in the appropriate degrees. To begin, we have

LeMmA 5.17. $Q^{2 i}(B)=Q^{2 i}\left(H^{*}\right)$ for $2 i \leqslant 2^{s+1}+2^{s}-4$.

Proof. First of all, the map $Q^{\text {even }}(B) \rightarrow Q^{\text {even }}\left(H^{*}\right)$ is surjective in degrees $\leqslant 2^{s+1}+2^{s}-4$. For, by our induction hypothesis, Theorem 5.1 holds in degree $<2^{s+1}+2^{s}-2$. Thus Corollary 5.2 holds in degree $<2^{s+1}+2^{s}-2$. Therefore, to prove surjectivity, it suffices to show that the $B(q)$ which appears in $B$ can be chosen with $q \geqslant 2^{s+1}-2$. By 5.13, $x$ can only be hit by the Steenrod powers $\mathrm{Sq}^{2^{2}-1}$. Since $\mathscr{A}(2)$ acts unstably we can also assume that $i \leqslant s$. So $x \notin B\left(2^{s+1}-2\right)$.

Second, the map $Q^{\text {even }}(B) \rightarrow Q^{\text {even }}\left(H^{*}\right)$ is injective in degrees $\leqslant 2^{s+1}+2^{s}-4$. Pick an indecomposable $b \in B^{\text {even }}$ of degree $<2^{s+1}+2^{s}-2$. Let $B^{\prime}$ be the sub-Hopf algebra of $B$ generated by the elements of degree $<|b|$. Thus $0 \neq b \in P\left(B / / B^{\prime}\right) \subset$ $P\left(H^{*} / / B^{\prime}\right)$. By 4.10 of [13] either $b$ is indecomposable in $H^{*} / / B^{\prime}$ or $b=a^{2}$. We can eliminate $b=a^{2}$. For our surjectivity argument shows that $B / / B^{\prime} \rightarrow H^{*} / / B^{\prime}$ is surjective in degree $<2^{s+1}+2^{s}-2$. So $b=a^{2}$ in $H^{*} / / B^{\prime}$ implies $b=a^{2}$ in $B / / B^{\prime}$. Q.E.D.

The inclusion $\xi B \subset \xi H^{*}$ induces a map $Q(\xi B) \rightarrow Q\left(\xi H^{*}\right)$.

Corollary 5.18. $Q^{4 i}(\xi B) \cong Q^{4 i}\left(\xi H^{*}\right)$ for $4 i \leqslant 2^{s+2}+2^{s+1}-8$.

Proof. Take a Borel decomposition $\otimes A_{i}$ of $H^{*}$ with generators $\left\{a_{i}\right\}$. Then the elements $\left\{a_{i}^{2}\right\}$ are generators of a Borel decomposition of $\xi H^{*}$. Q.E.D.

We can now prove

LEMMA 5.19. $Q^{4 i}(B)=0$ for $4 i \leqslant 2^{s+2}+2^{s+1}-8$.

Proof. Consider the commutative diagram:

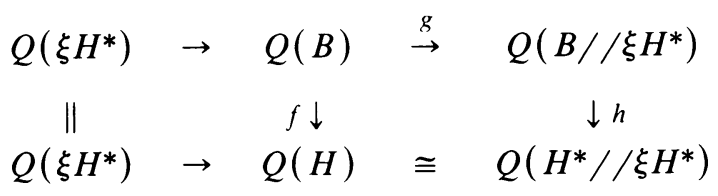

The rows are exact by 4.10 of [13]. Thus, by $5.18 \mathrm{~g}$ is injective in degrees $4 i \leqslant 2^{s+2}+2^{s+1}-8$. Since $B / / \xi H^{*} \rightarrow H^{*} / / \xi H^{*}$ is an injective map between exterior Hopf algebras it follows that $h$ is injective in all degrees. (Use an argument 
similar to that used to prove 5.17.) Thus $f$ is injective in degrees $4 i \leqslant 2^{s+2}+2^{s}-8$. Since $Q^{4 i}\left(H^{*}\right)=0$ for $i$ (see 2.1) the lemma follows. Q.E.D.

It follows from 5.19 that $\operatorname{Sq}^{\Delta_{t}}(y) \in \bar{B} \cdot \bar{B}$ for $1 \leqslant t \leqslant s$. So the secondary operation $\phi$ associated with (5.15) is defined on $y$ and satisfies $\bar{\mu}^{*} \phi(y)=x \otimes x+$ $\sum \mathrm{Im} \mathrm{Sq}^{4 n-2^{1+1}+4} \mathrm{Sq}^{1}$ in $H^{*} / / B \otimes H^{*} / / B$. For each $q>0$ we have

$$
\begin{aligned}
(\alpha \otimes \alpha) \mathrm{Sq}^{2 q} \mathrm{Sq}^{1} & =\sum_{i+j=2 q}\left(\alpha \mathrm{Sq}^{i} \otimes \alpha \mathrm{Sq}^{j}\right) \mathrm{Sq}^{1} \\
& =\sum_{i+j=2 q} \alpha \mathrm{Sq}^{i} \mathrm{Sq}^{1} \otimes \alpha \mathrm{Sq}_{q}^{j}+\alpha \mathrm{Sq}^{i} \otimes \alpha \mathrm{Sq}^{j} \mathrm{Sq}^{1}=0 .
\end{aligned}
$$

The first two identities follow from the Cartan formula. For the last equality $\alpha \mathrm{Sq}^{i}=\alpha \mathrm{Sq}^{j}=0$ if $i$ and $j$ are even by 5.13 while $\alpha \mathrm{Sq}^{i} \mathrm{Sq}^{1}=\alpha \mathrm{Sq}^{j} \mathrm{Sq}^{1}=0$ if $i$ and $j$ are odd by 2.5 .

Thus $\left\langle\alpha \otimes \alpha, \operatorname{Im~Sq}^{2 q} \mathrm{Sq}^{1}\right\rangle=0$ for each $q>0$. So $\alpha \otimes \alpha$ annihiliates every summand in $\bar{\mu}^{*} \phi(x)$ except for $x \otimes x$. It follows that $\left\langle\alpha^{2}, \phi(x)\right\rangle=\left\langle\alpha \otimes \alpha, \bar{\mu}^{*} \phi(x)\right\rangle=$ $\langle\alpha \otimes \alpha, x \otimes x\rangle \neq 0$. We are forced to conclude $\alpha^{2} \neq 0$, providing the desired contradiction to 2.4. Q.E.D.

(F) Corollaries 5.3 and 5.4. Assume, by induction, that 5.3 and 5.4 hold for $s^{\prime}<s$. We begin with Corollary 5.3 in the case $s$. Consider $\bar{x} \in \hat{Q}^{2^{s+1} k+2^{s}-1}$. By Theorem 5.1 we can write

$$
\bar{x}=\operatorname{Sq}^{2^{s} k}(\bar{y})+\operatorname{Sq}^{2^{s}}(\bar{z}),
$$

where $\bar{y} \in \hat{Q}^{2^{s} k+2^{s}-2}$ and $\bar{z} \in \hat{Q}^{2^{s} k-2}$. First of all,

$$
\mathrm{Sq}^{2^{s}} \mathrm{Sq}^{2^{s}}(\bar{x})=\sum_{i=0}^{s-1} \theta_{i}(\bar{z})=0
$$

where $\theta_{i} \in$ the ideal $\left(\mathrm{Sq}^{2^{i}}\right)^{2}$. The first equality follows from (3.10). For the second equality we use Corollary 5.4 for $s^{\prime}<s$ plus 4.5 . Second, if $k$ is odd, then

$$
\mathrm{Sq}^{2^{s}} \operatorname{Sq}^{2^{s} k}(\bar{y})=\sum_{i=0}^{s-1} \theta_{i}(\bar{y})=0
$$

by a similar argument. Finally, if $k$ is even, then

$$
\mathrm{Sq}^{2^{s}} \mathrm{Sq}^{2^{s} k}(\bar{y})=\mathrm{Sq}^{2^{s} k+2^{s}}(\bar{y})+\sum_{i=0}^{s-1} \theta_{i}(\bar{y})=0 .
$$

For the first equality use (3.11). In the second equality $\operatorname{Sq}^{2^{s} k+2^{s}}(\bar{y})=0$ since $\mathscr{A}(2)$ acts unstably.

Corollary 5.4 is an easy consequence of the Adem relations plus the fact that Corollary 5.3 holds for $s^{\prime} \leqslant s$.

6. Proof of Theorem 1.2, part II. Our next result about $\hat{Q}$ is easier to express if we dualize. Let $\hat{P}=$ the dual Steenrod module to $\hat{Q}$. We know from 5.1 that given $0 \neq \alpha \in \hat{P}_{2^{s+1} k+2^{s}-2}$, then either $\alpha \mathrm{Sq}^{2^{s}} \neq 0$ or $\alpha \mathrm{Sq}^{2^{s} k} \neq 0$. In this section we will prove

TheOREM 6.1. Given $0 \neq \alpha \in \hat{P}_{2^{t+1}+2^{s}-2}$, where $t>s$, then $\alpha \mathrm{Sq}^{2 k} \neq 0$ for $2 k>2^{s}$. 
The quotient map $\hat{Q} \rightarrow Q$ dualizes to give an inclusion $P \subset \hat{P}$. We can restrict to the case $\alpha \in P \subset \hat{P}$. For, by 5.7, $Q^{2^{t+1}+2^{s}-2} \xi H^{*} \subset \mathrm{Im} \mathrm{Sq}^{2^{t}}$. Thus it suffices to prove

TheOREM 6.2. Given $0 \neq \alpha \in P_{2^{t+1}+2^{s}-2}$, where $t>s \geqslant 2$, then $\alpha \mathrm{Sq}^{2 k} \neq 0$ for $2 k>2^{s}$.

Our argument for proving 6.2 is closely modelled on that from (E) of $\S 5$. Our proof is by contradiction. We will assume that

$$
\alpha \mathrm{Sq}^{2 k}=0 \text { for } 2 k>2^{s} \text {. }
$$

We will show that $\alpha^{2} \neq 0$ contradicting Lemma 2.4. First of all we have

Lemma 6.3. If $\operatorname{deg} \mathrm{Sq}^{R}=2 l>0$ and $\alpha \mathrm{Sq}^{R} \neq 0$, then $2 l=2^{s}$ and $\alpha \mathrm{Sq}^{R}=\alpha \mathrm{Sq}^{2}$.

Proof. We can write $\mathrm{Sq}^{R}$ as a polynomial in the operations $\left\{\mathrm{Sq}^{2^{i}}\right\}_{i \geqslant 0}$. None of the factors are $\mathrm{Sq}^{1}$. For since deg $\mathrm{Sq}^{R}$ is even any term from $\left(\mathrm{Sq}^{1}\right)$ actually belongs to $\left(\mathrm{Sq}^{1}\right)^{2}$. By 2.8 such terms act trivially on $\alpha$. Next if $\alpha \mathrm{Sq}^{2^{i 1}} \mathrm{Sq}^{2^{i 2}} \cdots \mathrm{Sq}^{2^{i r}} \neq 0$, then, by 5.4, $i_{1} \geqslant s$. Since $\alpha \mathrm{Sq}^{2 k}=0$ for $2 k>2^{s}$ we must have $i_{1}=s$. Since $\operatorname{deg} \alpha \mathrm{Sq}^{2^{s}}=$ $2^{t+1}-2$ it follows from 5.4 that $r=1$. Q.E.D.

LeMma 6.4. $\alpha \mathrm{Sq}^{k} \neq 0$ for some $k>2^{s}$.

Proof. Suppose

$$
\left\langle\alpha, F_{q} Q\right\rangle=0, \quad\left\langle\alpha, F_{q+1} Q\right\rangle \neq 0 .
$$

Pick $\bar{x} \in F_{q+1} \hat{Q}$ such that $\langle\alpha, \bar{x}\rangle \neq 0$. As in $\S 4$ let $B=B(q)$. Then $\langle\alpha, B\rangle=0$. Pick a representative $x \in H^{2^{1+1}+2^{s}-2}$ for $\bar{x}$ such that $\bar{\mu}^{*} x \in B \otimes B$. We will use the secondary operation associated with the relation

$$
\mathrm{Sq}^{2^{t+1}+2^{s}-1}=\sum_{1 \leqslant i \leqslant t} \mathrm{Sq}^{2^{t+1}+2^{s}-2^{t}} \mathrm{Sq}^{\Delta_{i}}+\mathrm{Sq}^{\Delta_{t+1}} \mathrm{Sq}^{2^{s}}
$$

By 4.4 and 4.5 we have $\mathrm{Sq}^{\Delta_{1}} x \in \bar{B} \cdot \bar{B}$. Moreover, by 4.4 and 5.3 , we have $\mathrm{Sq}^{2^{s}} x \in \bar{B} \cdot \bar{B}$. So the secondary operation associated to $(*)$ is defined on $x$ and satisfies

$$
\bar{\mu}^{*} \phi(x)=x \otimes x+\sum_{1 \leqslant i \leqslant t} \operatorname{Im~Sq} 2^{2^{+1}+2^{s}-2^{\prime}}+\operatorname{Im~Sq}^{\Delta_{t+1}}
$$

in $H^{*} / / B \otimes H^{*} / / B$. We have

$$
\alpha \mathrm{Sq}^{\Delta_{t+1}}=0 .
$$

For, dually, given $y \in Q^{2^{s}-1}$, we have

$$
\left\langle\alpha \mathrm{Sq}^{\Delta_{t+1}}, y\right\rangle=\left\langle\alpha, \mathrm{Sq}^{\Delta_{t+1}} y\right\rangle=0 .
$$

To establish the second equality observe, first of all, that

$$
\begin{aligned}
\mathrm{Sq}^{\Delta_{s}} y & =\sum_{i<0} \mathrm{Sq}^{2^{s}-2^{i}} \mathrm{Sq}^{\Delta_{i}} y=\mathrm{Sq}^{2^{s}-1} y \\
& =y^{2}=0
\end{aligned}
$$


(The equality $y^{2}=0$ follows from the fact that we are working in $Q$.) Hence

$$
\begin{aligned}
\mathrm{Sq}^{\Delta_{t+1}} y & =\mathrm{Sq}^{2^{s} \Delta_{t-s+1}} \mathrm{Sq}^{\Delta_{s}}(y)+\mathrm{Sq}^{\Delta_{s}} \mathrm{Sq}^{2^{s} \Delta_{t-s+1}}(y) \\
& =\mathrm{Sq}^{2^{s} \Delta_{t-s+1}} \mathrm{Sq}^{\Delta_{s}}(y) \quad(\mathscr{A}(2) \text { acts unstably }) \\
& =\mathrm{Sq}^{2^{s} \Delta_{t-s+1}}\left(y^{2}\right)=0 .
\end{aligned}
$$

(Again, the last equality depends on the fact that we are working on $Q$.)

Since $\alpha^{2}=0$ it follows from $\left({ }^{* *}\right)$ and $\left({ }^{* * *}\right)$ that we must have

$$
(\alpha \otimes \alpha) \mathrm{Sq}^{2^{t+1}+2^{s}-2^{i} \neq 0 \quad \text { for } i \leqslant t .}
$$

So $(\alpha \otimes \alpha) \mathrm{Sq}^{2 k} \neq 0$ for some $2 k \geqslant 2^{t}+2^{s}$. By the Cartan formula $\alpha \mathrm{Sq}^{k} \neq 0$ for $k \geqslant 2^{t-1}+2^{s-1}$. Since $t>s$ we have $k>2^{s}$. Q.E.D.

We now set about forcing our contradiction $\alpha^{2} \neq 0$. Our argument is an analogue of that used to prove 6.4. Replace the relation $\left({ }^{*}\right)$ used in the proof of 6.4 with

$$
\mathrm{Sq}^{2^{t+1}+2^{s}-1} \mathrm{Sq}^{1}=\sum_{2 \leqslant i \leqslant t}\left(\mathrm{Sq}^{2^{2+1}+2^{s}-2^{i}} \mathrm{Sq}^{1}\right) \mathrm{Sq}^{\Delta_{i}}+\left(\mathrm{Sq}^{\Delta_{t+1}}\right) \mathrm{Sq}^{2^{s}} \mathrm{Sq}^{1}
$$

The use of this relation demands a more subtle choice of a representative $x \in$ $H^{2^{t+1}+2^{s}-2}$ and of the sub-Hopf algebra $B \subset H^{*}$. Suppose

$$
\left\langle\alpha, F_{q} Q\right\rangle=0, \quad\left\langle\alpha, F_{q+1} Q\right\rangle \neq 0 .
$$

Pick $\bar{x} \in F_{q+1} Q$, where $\langle\alpha, \bar{x}\rangle=0$. Next choose $\bar{y} \in Q^{2^{t+1}+2^{s}-3}$, where $\mathrm{Sq}^{1} \bar{y}=\bar{x}$, $\bar{y} \in F_{q+1} Q$ and $\bar{y} \notin F_{q} Q$. We must justify such a choice. Write $\bar{x}=\mathrm{Sq}^{R} \bar{z}$, where $\operatorname{deg} \bar{z}=q+1$. It follows from Lemma 6.4 that $\operatorname{deg} \mathrm{Sq}^{R}>2^{s}$. It then follows from Lemma 6.3 that deg $\mathrm{Sq}^{R}$ is odd. So $\mathrm{Sq}^{R}=\mathrm{Sq}^{\Delta_{i}} \mathrm{Sq}^{R^{\prime}}$ for some $i \geqslant 1$. Thus

$$
\begin{aligned}
\bar{x} & =\mathrm{Sq}^{\Delta_{i}} \mathrm{Sq}^{R^{\prime}}(\bar{z})=\left(\mathrm{Sq}^{1} \mathrm{Sq}^{2 \Delta_{i-1}}+\mathrm{Sq}^{2 \Delta_{i-1}} \mathrm{Sq}^{1}\right) \mathrm{Sq}^{R^{\prime}}(\bar{z}) \\
& =\mathrm{Sq}^{1} \mathrm{Sq}^{2 \Delta_{i-1}} \mathrm{Sq}^{R^{\prime}(\bar{z}) .}
\end{aligned}
$$

For the last equality, observe that 6.2 forces $\mathrm{Sq}^{2 \Delta_{i-1}}=\mathrm{Sq}^{2^{s}}$. This is clearly impossible $\left(s \geqslant 2\right.$ !). So let $\bar{y}=\mathrm{Sq}^{2 \Delta_{i-1}} \mathrm{Sq}^{R^{\prime}}(\bar{z})$. The first two properties of $\bar{y}$ are immediate. The third property is forced by the fact that $\bar{x} \notin F_{q} Q$.

Let $B=$ the sub-Hopf algebra, invariant under $\mathscr{A}(2)$, generated by $B(q)$ and $\xi H^{*}$. Then $\langle\alpha, B\rangle=0$ and we can choose representatives $y$ and $x=\mathrm{Sq}^{1} y$ for $\bar{y}$ and $\bar{x}=\mathrm{Sq}^{1} \bar{y}$ so that $x \notin B$ and $\bar{\mu}^{*} y \in B \otimes B$. The operation $\phi$ associated to (6.5) is defined on $y$. First of all, by 5.3 and 4.4 we have

LemMa 6.6. $\mathrm{Sq}^{2^{s}} \mathrm{Sq}^{1} y \in \bar{B} \cdot \bar{B}$.

LeMma 6.7. $\mathrm{Sq}^{\Delta_{i}} y \in \bar{B} \cdot \bar{B}$ for $2 \leqslant i \leqslant t$.

To show Lemma 6.7 requires more work than for Lemma 6.6. The argument mimics those from 5.16-5.19. As in 5.16 we show

(a) $\mathrm{Sq}^{\Delta_{i}} y \in B$.

By analogues of the arguments in 5.17-5.19 we show

(b) $Q^{4 i} B=0$ for $4 i \leqslant 2^{t+1}+2^{t}+2^{s}-4$. 
Observe that deg $y=2^{t+1}+2^{s}-3$ and $\operatorname{deg} \mathrm{Sq}^{\Delta_{1}} \leqslant 2^{t}-1$. So $\operatorname{deg} \mathrm{Sq}^{\Delta_{1}} y \leqslant 2^{t+1}+$ $2^{t}+2^{s}-4$. Thus the above two results suffice to prove Lemma 6.7. In proving the above results there is only one point at which a new argument must be introduced to supplement those given in $§ 5$. When we prove (b) we first show

$$
Q^{2 i} B=Q^{2 i} H^{*} \text { for } 2 i \leqslant 2^{t}+2^{t-1}+2^{s-1}-2 .
$$

The proof follows that in 5.17. However, to apply that argument we need to know that the $B(q)$ which appears in $B$ can be chosen with $q \geqslant 2^{t}-2$. So we want to eliminate the possibility of $x=\mathrm{Sq}^{R} z$, where $\operatorname{deg~Sq}{ }^{R}>2^{t}+2^{s}$. By $6.3 \mathrm{Sq}^{R}$ has odd degree. So $\mathrm{Sq}^{R}=\mathrm{Sq}^{R^{\prime}} \mathrm{Sq}^{\Delta_{i}}$ for some $i$. By iterating (3.8) we can further reduce to the case $\mathrm{Sq}^{R}=\mathrm{Sq}^{R^{\prime}} \mathrm{Sq}^{\Delta}$, for some $i$. Then, by 6.3 , we can assume either

$$
\mathrm{Sq}^{R}=\mathrm{Sq}^{\Delta,} \quad(i \geqslant t+1),
$$

or

$$
\mathrm{Sq}^{R}=\mathrm{Sq}^{2^{s}} \mathrm{Sq}^{\Delta_{i}} \quad(i \geqslant t+1)
$$

$\mathrm{Sq}^{R}=\mathrm{Sq}^{\Delta_{i}}$ : We can write $\mathrm{Sq}^{\Delta_{i}}=\mathrm{Sq}^{\Delta_{i-1}} \mathrm{Sq}^{2^{i-1}}+\mathrm{Sq}^{2^{i-1}} \mathrm{Sq}^{\Delta_{i-1}}$. Since $i \geqslant t+1$ we can ignore the term $\mathrm{Sq}^{\Delta_{i-1}} \mathrm{Sq}^{2^{i-1}}$. For $\mathscr{A}(2)$ acts unstably on $H^{*}$. Likewise, by 6.3 and the fact that $i \geqslant t+1>s+1$, we can ignore the term $\mathrm{Sq}^{2^{i-1}} \mathrm{Sq}^{\Delta_{i-1}}$.

$\mathrm{Sq}^{R}=\mathrm{Sq}^{2^{s}} \mathrm{Sq}^{\Delta_{i}}$ : Since $\operatorname{deg} x=2^{t+1}+2^{s}-2$ and $i \geqslant t+1$ we must have $i=t$ +1 and $\operatorname{deg} z=1$. However $\mathscr{A}(2)$ acts trivially on $Q$ in degree 1 . This concludes the proof of 6.7 .

Because of Lemmas 6.6 and 6.7 the operation $\phi$ associated to (6.5) is defined on $y$ and we have

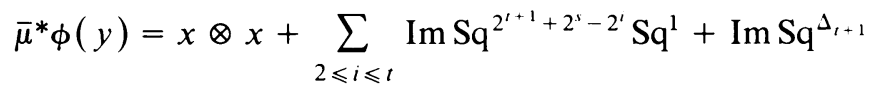

in $H^{*} / / B \otimes H^{*} / / B$. First of all

$$
(\alpha \otimes \alpha) \mathrm{Sq}^{\Delta_{t+1}}=\left(\alpha \mathrm{Sq}^{\Delta_{t+1}}\right) \otimes \alpha+\alpha \otimes\left(\alpha \mathrm{Sq}^{\Delta_{t+1}}\right)=0 .
$$

To establish the last equality we use the identities

$$
\alpha \mathrm{Sq}^{\Delta_{t+1}}=\alpha\left(\mathrm{Sq}^{\Delta_{t}} \mathrm{Sq}^{2^{t}}+\mathrm{Sq}^{2^{t}} \mathrm{Sq}^{\Delta_{t}}\right)=0 .
$$

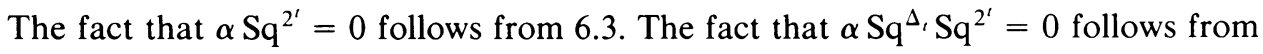
the fact that $\mathscr{A}(2)$ acts unstably on $H^{*}$.

Secondly, for each $2 q>2^{s+1}$ we have

$$
\begin{aligned}
(\alpha \otimes \alpha) \mathrm{Sq}^{2 q} \mathrm{Sq}^{1} & =\sum_{i+j=2 q}\left(\alpha \mathrm{Sq}^{i} \otimes \alpha \mathrm{Sq}^{j}\right) \mathrm{Sq}^{1} \\
& =\sum_{i+j=2 q} \alpha \mathrm{Sq}^{i} \mathrm{Sq}^{1} \otimes \alpha \mathrm{Sq}^{j}+\alpha \mathrm{Sq}^{i} \otimes \alpha \mathrm{Sq}^{j} \mathrm{Sq}^{1}=0
\end{aligned}
$$

For the last equality $\alpha \mathrm{Sq}^{i}=\alpha \mathrm{Sq}^{j}=0$ if $i$ and $j$ are even by 6.3 while $\alpha \mathrm{Sq}^{i} \mathrm{Sq}=$ $\alpha \mathrm{Sq}^{j} \mathrm{Sq}=0$ if $i$ and $j$ are odd by 2.3 .

Thus $\alpha \otimes \alpha$ annihilates every summand in $\bar{\mu}^{*} \phi(y)$ except for $x \otimes x$. It follows that

$$
\left\langle\alpha^{2}, \phi(y)\right\rangle=\left\langle\alpha \otimes \alpha, \bar{\mu}^{*} \phi(y)\right\rangle=\langle\alpha \otimes \alpha, x \otimes x\rangle \neq 0 .
$$

This forces $\alpha^{2} \neq 0$ providing the desired contradiction to 2.4 . 
7. Relations in $\mathscr{A}(2)$. In $\S 8$ we will use Theorems 5.1 and 6.1 to prove Theorem 1.2. In this section, as a preliminary, we deduce further facts about the mod 2 Steenrod algebra $\mathscr{A}(2)$. We can reduce elements of $\mathscr{A}(2)$ to polynomials in the operations $\left\{\mathrm{Sq}^{2^{i}}\right\}_{i \geqslant 0}$. In [16] Wall described $\mathscr{A}(2)$ from this viewpoint. Wall analyzed the relations in $\mathscr{A}(2)$ via the concept of height. We will use a different approach. However, the results obtained are obviously based on those in [16].

We will analyze $\mathscr{A}(2)$ via the augmentation fibration. The augmentation filtration on $\mathscr{A}(2)$ is defined by:

$$
F_{0}=\mathscr{A}(2), \quad F_{n}=\operatorname{Image}\left\{\phi_{(n)}: \bigotimes_{i=1}^{n} \overline{\mathscr{A}(2)} \rightarrow \mathscr{A}(2)\right\} .
$$

Here $\phi_{(n)}$ is defined recursively by $\phi_{(n+1)}=\phi\left(\phi_{(n)} \otimes 1\right)$, where $\phi: \mathscr{A}(2) \otimes \mathscr{A}(2) \rightarrow$ $\mathscr{A}(2)$ is multiplication on $\mathscr{A}(2)$. Thus $F_{n}=$ the $n$ fold decomposables of $\mathscr{A}(2)$.

(A) Relations in $\mathscr{A}(2)$. We first show that the following relations hold in $\mathscr{A}(2)$.

Relation 7.1. $\mathrm{Sq}^{2^{i}} \mathrm{Sq}^{2 j}+\mathrm{Sq}^{2 j} \mathrm{Sq}^{2^{i}} \equiv 0 \bmod F_{4}(0 \leqslant j \leqslant i-3)$.

Relation 7.2. $\mathrm{Sq}^{2^{i}} \mathrm{Sq}^{2^{i-2}}+\mathrm{Sq}^{i^{i-2}} \mathrm{Sq}^{2^{i}} \equiv \mathrm{Sq}^{2^{i-1}} \mathrm{Sq}^{2^{i-2}} \mathrm{Sq}^{2^{i-1}} \bmod F_{4}(i \geqslant 2)$.

Relation 7.3. $\mathrm{Sq}^{2^{i}} \mathrm{Sq}^{2^{i}} \equiv \mathrm{Sq}^{2^{i-1}} \mathrm{Sq}^{2^{i}} \mathrm{Sq}^{2^{i-1}} \bmod F_{4}$.

Proof of 7.1. It suffices to show that the relation holds modulo the ideal $\left(\mathrm{Sq}^{1}, \mathrm{Sq}^{2}, \ldots, \mathrm{Sq}^{2^{j-2}}\right)$. For any element of $\left(\mathrm{Sq}^{1}, \mathrm{Sq}^{2}, \ldots, \mathrm{Sq}^{2^{j-2}}\right)$ of degree $2^{i}+2^{j}$ belongs to $F_{4}$. We have an isomorphism

$$
\mathscr{A}(2) /\left(\mathrm{Sq}^{1}, \ldots, \mathrm{Sq}^{2^{j-2}}\right) \cong \mathscr{A}(2)
$$

of Hopf algebras, where $\mathrm{Sq}^{2^{k}} \in \mathscr{A}(2) /\left(\mathrm{Sq}^{1}, \ldots, \mathrm{Sq}^{2^{j-2}}\right)$ corresponds to $\mathrm{Sq}^{2^{k-j+2}} \in$ $\mathscr{A}(2)$. So it suffices to show the relations

$$
\mathrm{Sq}^{2^{k}} \mathrm{Sq}^{2}+\mathrm{Sq}^{2} \mathrm{Sq}^{2^{k}} \equiv 0 \quad \bmod F_{4}, k \geqslant 4,
$$

hold in $\mathscr{A}(2)$. Using Milnor's rule of multiplication from [12] we have

$$
\mathrm{Sq}^{2^{k}} \mathrm{Sq}^{2}+\mathrm{Sq}^{2} \mathrm{Sq}^{2^{k}}=\mathrm{Sq}^{2^{k}-4,2} \equiv 0 \bmod F_{4}
$$

Proof of 7.2. As above, we can work modulo the ideal $\left(\mathrm{Sq}^{1}, \mathrm{Sq}^{2}, \ldots, \mathrm{Sq}^{2^{i+1}}\right)$. So it suffices to show

$$
\mathrm{Sq}^{8} \mathrm{Sq}^{2}+\mathrm{Sq}^{2} \mathrm{Sq}^{8} \equiv \mathrm{Sq}^{4} \mathrm{Sq}^{2} \mathrm{Sq}^{4} \bmod F_{4}
$$

Using the rule of multiplication in [12] we have

$$
\mathrm{Sq}^{8} \mathrm{Sq}^{2}+\mathrm{Sq}^{2} \mathrm{Sq}^{8}=\mathrm{Sq}^{4,2}
$$

while

$$
\mathrm{Sq}^{4} \mathrm{Sq}^{2} \mathrm{Sq}^{4} \equiv \mathrm{Sq}^{4} \mathrm{Sq}^{6} \equiv \mathrm{Sq}^{4,2} \quad \operatorname{Mod} F_{4}
$$

Proof OF 7.3. As in $\S 3$ of [16] we can use Milnor's rule of multiplication to show that 7.3 holds modulo an error term belonging to the algebra $\mathscr{S}(i-1)$ generated by $\left\{\mathrm{Sq}^{1}, \mathrm{Sq}^{2}, \ldots, \mathrm{Sq}^{2^{i-1}}\right\}$. In degree $2^{i+1}$ elements of $\mathscr{S}(i-1)$ belong to $F_{4}$.

(B) Allowable monomials. If $k \geqslant l$ let

$$
\theta_{l}^{k}=\mathrm{Sq}^{2^{k}} \mathrm{Sq}^{2^{k-1}} \cdots \mathrm{Sq}^{2^{l+1}} \mathrm{Sq}^{2^{l}}
$$


The monomial $\theta_{l_{m}}^{k} \cdots \theta_{l_{2}}^{k} \theta_{l_{1}}^{k}$ is $k$-allowable if $l_{m}<l_{m-1}<\cdots<l_{2}<l_{1}$. An allowable monomial is a product $M_{0} M_{1} \cdots M_{1} \cdots$, where $M_{i}$ is an $i$-allowable monomial and all but a finite number of the $M_{i}$ are empty products. We will show that the allowable monomials are a basis of $\mathscr{A}(2)$. Actually, we will prove a stronger result. To simplify notation let $A=\mathscr{A}(2)$. Let $E_{0} A$ be the associated graded Hopf algebra obtained from the augmentation filtration on $A$. The rest of the section will be spent in proving

THEOREM 7.4. The allowable monomials are a basis of $E_{0} A$.

(C) The Lie algebra L. Now $E_{0} A$ is a primitively generated Hopf algebra (see [13]). Let $L=P E_{0} A$, the module of primitives. We can define a restricted Lie algebra structure on $L$ via the two maps

$$
\begin{gathered}
{[,]: L \times L \rightarrow L, \quad[x, y]=x y-y x,} \\
\xi: L \rightarrow L, \quad \xi x=x^{2} .
\end{gathered}
$$

Then $E_{0} A=U(L)$, the universal enveloping Hopf algebra of $L$ (see [13]). So, to describe $E_{0} A$ it suffices to describe $L$. Let

$$
\begin{aligned}
& S_{i}^{i}=\mathrm{Sq}^{2^{i}}, \\
& S_{j}^{i}=\left[\left[\cdots\left[\mathrm{Sq}^{2^{i}}, \mathrm{Sq}^{2^{\prime-1}}\right] \cdots \mathrm{Sq}^{2^{\prime+1}}\right] \mathrm{Sq}^{2^{j}}\right] \quad(i>j) .
\end{aligned}
$$

The following three results completely describe the restricted Lie algebra structure of $L$.

Proposition 7.5. $\left\{S_{j}^{i} \mid i \geqslant j\right\}$ is a basis of $L$.

Proposition 7.6. If $i \geqslant k$, then

$$
\left[S_{j}^{i}, S_{l}^{k}\right]= \begin{cases}S_{l}^{i}, & j=k+1 \\ 0, & \text { otherwise }\end{cases}
$$

Proposition 7.7. $\left(S_{j}^{i}\right)^{2}=0$.

To prove these results we consider the dual Hopf algebra $A_{*}=\mathbf{Z} / 2\left[\xi_{1}, \xi_{2}, \ldots\right]$ of $A$. The augmentation filtratiuon $\left\{F_{q}\right\}$ on $A$ has a dual filtration defined by

$$
\begin{aligned}
& F_{0}=A, \\
& F^{n}=\operatorname{Ker}\left\{\phi^{(n)}: A_{*} \rightarrow \bigotimes_{i=1}^{n} A_{*}\right\} .
\end{aligned}
$$

Here $\phi^{(n)}$ is defined recursively by $\phi^{(n+1)}=\left(\phi^{(n)} \otimes 1\right) \phi_{*}$, where $\phi_{*}: A_{*} \rightarrow A_{*} \otimes A_{*}$ is comultiplication. The associated graded Hopf algebra ${ }_{0} E A_{*}$ is the dual Hopf algebra of $E_{0} A$ (see $[2,1.3]$ ). The Hopf algebra structure of ${ }_{0} E A_{*}$ can be derived from that of $A_{*}$. If $\left\{x_{1}, x_{2}, \ldots\right\}$ is a set of polynomial generators of $A_{*}$ (i.e. $\left.A_{*}=\mathbf{Z} / 2\left[x_{1}, x_{2}, \ldots\right]\right)$, then ${ }_{0} E A_{*}$ is an exterior algebra on the set of $\left\{x_{i}^{2 \prime} \mid i \geqslant 1\right.$, $j \geqslant 0\}$ (see [2, Theorem 1.2] as well as the discussion leading up to it). So let $e_{l}^{k}=\xi_{l}^{2^{k}}$. Then

LEMMA 7.8. ${ }_{0} E A_{*}$ is an exterior algebra on the set $\left\{e_{l}^{k} \mid k \geqslant 0, l \geqslant 1\right\}$. 
As for the coalgebra structure of ${ }_{0} E A_{*}$, the set $\left\{\xi_{l}^{2^{k}}\right\}$ satisfies the identity $\phi_{*} \xi_{l}^{2^{k}}=\sum_{i+j=l} \xi_{i}^{2^{k+j}} \otimes \xi_{j}^{2^{l}}$. So

LEMMA 7.9. $\phi_{*} e_{l}^{k}=\sum_{i+j=l} e_{i}^{k+j} \otimes e_{j}^{k}$.

In particular

LEMMA 7.10. $\phi^{(l)} e_{l}^{k}=e_{1}^{k+l-1} \otimes \cdots \otimes e_{1}^{k+1} \otimes e_{1}^{k}$.

Proof of Proposition 7.5. The pairing between $E_{0} A$ and ${ }_{0} E A_{*}$ induces a pairing between $L=P E_{0} A_{*}$ and $Q_{0} E A^{*}$, the module of indecomposables for ${ }_{0} E A_{*}$. We prove 7.5 by showing that the bases $\left\{e_{l}^{k}\right\}$ of $Q_{0} E A_{*}$ dualize to give $\left\{S_{j}^{i}\right\}$ as a basis of $l$. $A$ 1-1 pairing is provided by

LEMMA 7.5.1.

$$
\left\langle S_{j}^{i}, e_{l}^{k}\right\rangle= \begin{cases}1, & k=j, l=i-j+1, \\ 0, & \text { otherwise. }\end{cases}
$$

Proof. Since degree $S_{j}^{i}=2^{i+1}-2^{j}$ while $\operatorname{deg} e_{l}^{k}=2^{k+l}-2^{k}$ we have

$$
\left\langle S_{j}^{i}, e_{j}^{k}=0\right\rangle \quad \text { unless } k+l=i+1, k=j .
$$

So we are left with showing $\left\langle S_{k}^{k+l-1}, e_{l}^{k}\right\rangle \neq 0$. We can write $S_{k}^{k+l-1}$ as a sum, $\sum \mathrm{Sq}^{2^{i}} \mathrm{Sq}^{2^{i 2}} \cdots \mathrm{Sq}^{2^{i k}}$, of various permutations of $\mathrm{Sq}^{2^{k+1-1}} \cdots \mathrm{Sq}^{2^{k+1}} \mathrm{Sq}^{2^{k}}$ (including $\mathrm{Sq}^{2^{k+1-1}} \cdots \mathrm{Sq}^{2^{k}}$ itself). So

$$
\begin{aligned}
\left\langle S_{k}^{k+l-1}, e_{l}^{k}\right\rangle & =\left\langle\sum \mathrm{Sq}^{2^{i_{1}}} \cdots \mathrm{Sq}^{2^{i \prime}}, e_{l}^{k}\right\rangle \\
& =\sum\left\langle\phi_{(k)} \mathrm{Sq}^{2^{i_{1}}} \otimes \cdots \otimes \mathrm{Sq}^{2^{i_{l}}}, e_{l}^{k}\right\rangle \\
& =\sum\left\langle\mathrm{Sq}^{2^{i_{1}}} \otimes \cdots \otimes \mathrm{Sq}^{2^{\prime \prime}}, \phi^{(k)} e_{l}^{k}\right\rangle \\
& =\sum\left\langle\mathrm{Sq}^{2^{i_{1}}} \otimes \cdots \otimes \mathrm{Sq}^{2^{i_{l}}}, e_{1}^{k+l-1} \otimes \cdots \otimes e_{1}^{k}\right\rangle \\
& =\left\langle\mathrm{Sq}^{2^{k+1-1}} \otimes \cdots \otimes \mathrm{Sq}^{2^{k}}, e_{1}^{k+l-1} \otimes \cdots \otimes e_{1}^{k}\right\rangle .
\end{aligned}
$$

This last equality follows from (*). Moreover, since $e_{1}^{i}=\xi_{1}^{2^{i}}$ we have

$$
\left\langle\mathrm{Sq}^{2^{k+1-1}} \otimes \cdots \otimes \mathrm{Sq}^{2^{k}}, \quad e_{1}^{k+l-1} \otimes \cdots \otimes e_{1}^{k}\right\rangle \neq 0
$$

Proof of Proposition 7.6. Since $\left\{S_{j}^{i}\right\}$ is a basis, $L=0$ except in degrees of the form $2^{i+1}-2^{j}(i \geqslant j)$. Degree $\left[S_{j}^{i}, S_{l}^{k}\right]=2^{i+1}-2^{j}+2^{k+1}-2^{l}$. So $\left[S_{j}^{i}, S_{l}^{k}\right] \neq 0$ restricts us to the following possibilities:

(i) $j=k+1$ and $\left[S_{k+1}^{i}, S_{l}^{k}\right]=S_{k}^{i}$.

(ii) $j=k=l$ and $\left[S_{j}^{i}, S_{j}^{i}\right]=\mathrm{Sq}^{2^{i+1}}$.

(iii) $i=k, j=l$ and $\left[S_{j}^{i}, S_{j}^{i}\right]=S_{j+1}^{i+1}$.

The last two possibilities can easily be eliminated. For (ii) would force $\mathrm{Sq}^{2^{i+1}}$ to be decomposable while (iii) contradicts the fact that $[x, x]=0$ for all $x \in L$. The first possibility, on the other hand, always happens. That is

LEMMA 7.6. 1. $\left[S_{j}^{i}, S_{l}^{k}\right]=S_{l}^{i}$ whenever $j=k+1$. 
ProOF.

$$
\begin{aligned}
\left\langle\left[S_{k+1}^{i}, S_{l}^{k}\right], e_{i-l+1}^{l}\right\rangle & =\left\langle S_{k+1}^{i} S_{l}^{k}+S_{l}^{k} S_{l+1}^{i}, e_{i-l+1}^{l}\right\rangle \\
& =\left\langle\phi\left(S_{k+1}^{i} \otimes S_{l}^{k}+S_{l}^{k} \otimes S_{k+1}^{i}\right), e_{i-l+1}^{l}\right\rangle \\
& =\left\langle S_{k+1}^{i} \otimes S_{l}^{k}+S_{l}^{k} \otimes S_{k+1}^{i}, \phi_{*} e_{i-l+1}^{l}\right\rangle \\
& =\left\langle S_{k+1}^{i} \otimes S_{l}^{k}+S_{l}^{k} \otimes S_{k+1}^{i}, \sum_{s+t=i-l+1} e_{s}^{l+t} \otimes e_{t}^{l}\right\rangle \\
& =\left\langle S_{k+1}^{i} \otimes S_{l}^{k}, e_{i-k}^{k+1} \otimes e_{k-l+1}^{l}\right\rangle \neq 0 .
\end{aligned}
$$

The lemma now follows from 7.5.1. Q.E.D.

Proof of Proposition 7.7. If $\left(S_{j}^{i}\right)^{2} \neq 0$, then $\left\langle\left(S_{j}^{i}\right), e_{l}^{k}\right\rangle \neq 0$ for some $e_{l}^{k}$. But

$$
\left\langle\left(S_{j}^{i}\right), e_{l}^{k}\right\rangle=\left\langle\phi\left(S_{j}^{i} \otimes_{j}^{i}\right), e_{l}^{k}\right\rangle=\left\langle S_{j}^{i} \otimes S_{j}^{i}, \phi_{*} e_{l}^{k}\right\rangle=0 .
$$

The last equality follows from the fact that $\pi_{*} e_{l}^{k}$ does not contain $e_{i+j-1}^{j} \otimes e_{i+j-1}^{j}$ as a summand (see 7.9).

(D) Proof of Theorem 7.4. Before proving Theorem 7.4 let us first note some relations between the allowable monomials $\theta_{l}^{k}$ and the commutators $S_{l}^{k}$. Let

$$
\mathscr{S}(n)=\text { the subalgebra of } E_{0} A \text { generated by }\left\{\mathrm{Sq}^{1}, \mathrm{Sq}^{2}, \ldots, \mathrm{Sq}^{2^{n}}\right\} \text {. }
$$

LEMMA 7.11. $\theta_{l}^{k}=S_{l}^{k}+\sum m_{i}^{\prime} \mathrm{Sq}^{2^{k}} m_{i}^{\prime \prime}$, where $m_{i}^{\prime}, m_{i}^{\prime \prime} \in \mathscr{S}(n)$ and $\operatorname{deg} m_{i}^{\prime}>0$.

LEMMA 7.12. $\left(\theta_{l}^{k}\right)^{2}=\sum m_{i}^{\prime} \mathrm{Sq}^{2^{k}} m_{i}^{\prime \prime} \mathrm{Sq}^{2^{k}} m_{i}^{\prime \prime \prime}$, where $m_{i}^{\prime}, m_{i}^{\prime \prime}, m_{i}^{\prime \prime \prime} \in \mathscr{S}(n)$ and $\operatorname{deg} m_{i}^{\prime}>0$.

The first lemma follows from the definition of $S_{l}^{k}$. Regarding the second relation we have the identities

$$
\begin{aligned}
& 0=\left(S_{l}^{k}\right)^{2} \\
& =\theta_{l}^{k} S_{l}^{k}+\sum m_{j}^{\prime} \mathrm{Sq}^{2^{k}} m_{j}^{\prime \prime} \mathrm{Sq}^{2^{k}} \quad(\text { by 7.11) } \\
& =S_{l}^{k} \theta_{l}^{k}+\sum m_{j}^{\prime} \mathrm{Sq}^{2^{k}} m_{j}^{\prime \prime} \mathrm{Sq}^{2^{k}} m_{j}^{\prime \prime \prime} \quad(\text { by 7.6) } \\
& =\left(\theta_{l}^{k}\right)^{2}+\sum m_{i}^{\prime} \mathrm{Sq}^{2^{k}} m_{i}^{\prime \prime} \mathrm{Sq}^{2^{k}} m_{i}^{\prime \prime \prime} \quad(\text { by 7.11). }
\end{aligned}
$$

To prove 7.4 it suffices to prove that the allowable monomials are a spanning set of $E_{0} A$. Let

$$
\begin{aligned}
& M_{n}=\text { the rank of } E_{0} A \text { in degree } n(\text { as a } \mathbf{Z} / 2 \text { vector space), } \\
& N_{n}=\text { the number of allowable monomials of degree } n .
\end{aligned}
$$

LEMMA 7.13. $M_{n}=N_{n}$ for all $n$.

Proof. We have the identities

$$
\sum_{n \geqslant 0} M_{n} t^{n}=\prod_{0 \leqslant j \leqslant i}\left(1+t^{2^{1+1}-2^{j}}\right)=\sum_{n \geqslant 0} N_{n} t^{n} .
$$

For the first equality think of $M_{n}$ as being the rank of ${ }_{0} E A_{*}$ in degree $n$. Since ${ }_{0} E A_{*}$ is an exterior algebra on the elements $\left\{e_{j}^{i}\right\}$, the Poincaré series of ${ }_{0} E A_{*}$ is 
$\Pi_{0 \leqslant j \leqslant i}\left(1+t^{2^{i+1}-2^{\prime}}\right)$. Regarding the second equality the Poincaré series $\sum_{n \geqslant 0} N_{n} t^{n}$ of allowable monomials is the same as that of an exterior algebra on the symbols $\left\{\theta_{j}^{i}\right\}$. So, again $\sum N_{n} t^{n}=\prod_{0 \leqslant j \leqslant i}\left(1+t^{2^{L+1}-2^{j}}\right)$. Q.E.D.

Our proof of 7.4 will be by induction. We will show that for each $n \geqslant 0$

(*) the allowable monomials in $\left\{\mathrm{Sq}^{1}, \ldots, \mathrm{Sq}^{2^{n}}\right\}$ form a spanning set for $\mathscr{S}(n)$.

To prove $(*)$ for $\mathscr{S}(n)$ we will require the following result:

LEMMA 7.14. If $m_{1}, m_{2} \in \mathscr{S}(n-1)$, then $m_{1} \mathrm{Sq}^{2^{n}} m_{2}=\sum_{1 \leqslant i \leqslant n} m_{i}^{\prime} \theta_{i}^{n}$ for some $m_{i}^{\prime} \in \mathscr{S}(n-1)$.

Proof. We can assume $m_{1}$ and $m_{2}$ are in allowable form. We will prove 7.14 by an inductive argument based on $\operatorname{deg} m_{2}$. If $\operatorname{deg} m_{2}=0$, then 7.14 holds trivially. Suppose it is true provided $\operatorname{deg} m_{2}<k$. Let $\operatorname{deg} m_{2}=k$. Write $m_{2}=\mathrm{Sq}^{2^{i}} m_{2}^{\prime}$. If $i \leqslant n-2$, then

$$
m_{1} \mathrm{Sq}^{2^{n}} m_{2}=m_{1} \mathrm{Sq}^{2^{n}} \mathrm{Sq}^{2^{i}} m_{2}^{\prime}=m_{1} \mathrm{Sq}^{2^{i}} \mathrm{Sq}^{2^{n}} \mathrm{Sq}^{\prime}{ }_{2} \quad \text { (by 7.6) }
$$

and we have reduced the degree of $m_{2}$. So suppose $i=n-1$. Either $m_{2}=\theta_{j}^{n-1}$ whence $m_{1} \mathrm{Sq}^{2^{n}} m_{2}=m_{1} \theta_{j}^{n}$, or $m_{2}=\theta_{j}^{n-1} \mathrm{Sq}^{2^{n-1}} m_{2}^{\prime \prime}$. In this latter case

$$
\begin{aligned}
\mathrm{Sq}^{2^{n}} m_{2} & =\mathrm{Sq}^{2^{n}} \theta_{j}^{n} \mathrm{Sq}^{2^{n-1}} m_{2} \\
& =S_{j}^{n} \mathrm{Sq}^{2^{n-1}} m_{2}+\sum m_{j}^{\prime} \mathrm{Sq}^{2^{n}} m_{j}^{\prime \prime} \quad(\text { by } 7.11) \\
& \left.=\mathrm{Sq}^{2^{n-1}} S_{j}^{n} m_{2}+\sum m_{j}^{\prime} \mathrm{Sq}^{2^{n}} m_{j}^{\prime \prime} \quad \text { (by } 7.6\right) \\
& =\sum m_{i}^{\prime} \mathrm{Sq}^{2^{n}} m_{i}^{\prime \prime},
\end{aligned}
$$

where $\operatorname{deg} m_{i}^{\prime}>0$ and $\operatorname{deg} m_{i}^{\prime \prime}<k$. So we have again reduced the degree of $m_{2}$. Q.E.D.

We can extend 7.14 to

LEMMA 7.15. If $m_{1}, m_{2}, \ldots, m_{k+1} \in \mathscr{S}(n-1)$, then

$$
m_{1} \mathrm{Sq}^{2^{n}} m_{2} \cdots m_{k} \mathrm{Sq}^{2^{n}} m_{k+1}=\sum m_{i}^{\prime} \theta_{i_{1}}^{n} \theta_{i_{2}}^{n} \cdots \theta_{i_{k}}^{n},
$$

where $m_{i}^{\prime} \in \mathscr{S}(n-1)$.

Proof. By induction. The initial case is 7.14. By induction we can write

$$
m_{1} \mathrm{Sq}^{2^{n}} m_{2} \cdots m_{k} \mathrm{Sq}^{2^{n}} m_{k+1}=\sum m_{1} \mathrm{Sq}^{2^{n}} m_{i}^{\prime} \theta_{i_{2}}^{n} \cdots \theta_{i_{k}}^{n} .
$$

Apply 7.14 to $m_{1} \mathrm{Sq}^{2^{n}} m_{i}^{\prime}$ and we have reduced $m_{1} \mathrm{Sq}^{2^{n}} m_{2} \cdots m_{k} \mathrm{Sq}^{2^{n}} m_{k+1}$ to the desired form. Q.E.D.

We now prove (*) for $\mathscr{S}(n)$. Given a monomial $M=m_{1} \mathrm{Sq}^{2^{n}} m_{2} \cdots m_{k} \mathrm{Sq}^{2^{n}} m_{k+1}$ let $D=\left(d_{1}, d_{2}, \ldots, d_{k+1}, 0,0, \ldots\right)$, where $d_{i}=\operatorname{deg} m_{i}$. Order the sequences $\{D\}$ lexicographically. We will proceed by induction on this ordering. We can assume, by 7.15 that $M$ is of the form

$$
M=m \theta_{i_{1}}^{n} \cdots \theta_{i_{k}}^{n}, \quad m \in \mathscr{S}(n-1) .
$$

Since $m$ can be assumed to be in allowable form, $M$ will be in allowable form provided $i_{1}<i_{2}<\cdots<i_{k}$. If $i_{r+1} \geqslant i_{r}$ for some $r$, then $M$ contains $\left(\theta_{i_{r}}^{n}\right)^{2}$. But, by 
7.12, we can replace $\left(\theta_{i_{r}}^{n}\right)^{2}$ by $\sum m_{i}^{\prime} \mathrm{Sq}^{2^{n}} m_{i}^{\prime \prime} \mathrm{Sq}^{2^{\prime \prime}} m_{i}^{\prime \prime \prime}$, where $m_{i}^{\prime}, m_{i}^{\prime \prime}, m_{i}^{\prime \prime \prime} \in \mathscr{S}(n-1)$ and $\operatorname{deg} m_{i}^{\prime}>0$. Such a replacement lowers $D$. So we can assume that $M$ can be reduced to allowable form in this case as well.

8. Proof of Theorem 1.2. We will prove the dual of Theorem 1.2. As in $\S 6$ let $\hat{P}$ be the dual Steenrod module of $\hat{Q}$. So $\mathscr{A}(2)$ acts on the right of $\hat{P}$ and lowers degree. We want to prove

THEOREM 8.1. (a) If $2^{s} \in 2 n$, then $\mathrm{Sq}^{2^{s}}=0$ on $\hat{P}_{2 n}$.

(b) If $2^{s+1} \in 2 n$ and $2^{s} \notin 2 n$, then $\mathrm{Sq}^{2^{s}}: \hat{P}_{2 n} \rightarrow \hat{P}_{n-2^{s}}$ is injective.

The rest of $\S 8$ will be devoted to the proof of 8.1 .

(A) Reduction in proof of Theorem 8.1. Pick $0 \neq \alpha \in \hat{P}_{2 n}$. Let $\theta$ be an element of $\mathscr{A}^{\text {even }}(2)$ of maximal even degree such that $\alpha \theta \neq 0$. We can assume $\theta$ is a monomial in the operations $\left\{\mathrm{Sq}^{2^{\prime}}\right\}$. If $\theta=\mathrm{Sq}^{i^{1}} \cdots \mathrm{Sq}^{2^{2 \prime}}$ we say $\theta$ is of length $l$. If there is more than one choice for $\theta$, then choose $\theta$ to have maximal length. Since $\theta$ is of maximal length we can treat Relations 7.1-7.3 as identities when we manipulate the factors $\left\{\mathrm{Sq}^{2^{\prime}}\right\}$ appearing in $\theta$. We will use:

Rule 8.2. $\mathrm{Sq}^{2^{\prime}} \mathrm{Sq}^{2 \prime}=\mathrm{Sq}^{2^{j}} \mathrm{Sq}^{2^{\prime}}$ if $|i-j| \geqslant 2$.

Rule 8.3. $\mathrm{Sq}^{2^{i+1}} \mathrm{Sq}^{2^{i}} \mathrm{Sq}^{2^{i+1}}=\mathrm{Sq}^{2^{i}} \mathrm{Sq}^{2^{i+2}}+\mathrm{Sq}^{2^{i+2}} \mathrm{Sq}^{2^{i}}$.

Rule 8.4. $\mathrm{Sq}^{2^{i}} \mathrm{Sq}^{2^{\prime+1}} \mathrm{Sq}^{2^{i}}=\left(\mathrm{Sq}^{2^{i+1}}\right)^{2}$.

Rule 8.5. $\left(\mathrm{Sq}^{2^{i}}\right)^{2}=0$.

We can use these rules to replace any occurrence of the left-hand factor in $\theta$ by the right-hand factor.

By Theorem 7.4, we can assume that $\theta$ is an allowable monomial, $\theta=\theta_{l_{m}}^{k_{m}} \cdots \theta_{l_{1}}^{k_{1}}$. By Corollary $5.2 \operatorname{deg} \alpha \theta=2^{r+1}-2$ for some $r \geqslant 1$. We will prove

PROPOSITION 8.6 (a) $l_{i}=r+1-i$.

(b) $k_{m}<k_{m-1}<\cdots<k_{2}<k_{1}$.

First of all, however, we will show that Proposition 8.6 suffices to prove Theorem 8.1. Observe that it follows from 8.6 that

$$
2 n=2^{k_{1}+1}+2^{k_{2}+1}+\cdots+2^{k_{m}+1}+2^{r+1-m}-2 .
$$

So, if we define $\lambda(k)$ to be the number of terms in the binary expansion of $k$, then $(* *)$

$$
\lambda(2 n)=r .
$$

Corollary 8.7. $\alpha \mathrm{Sq}^{2^{s}}=0$ if $2^{s} \in 2 n$.

Proof. Suppose $2^{s} \in 2 n$ but $\beta=\alpha \mathrm{Sq}^{2^{s}} \neq 0$. Let $2 k=\operatorname{deg} \beta$. Then $\lambda(2 k)=$ $\lambda(2 n)-1=r-1$. By applying 8.6 we know that there exists $\psi \in \mathscr{A}^{\text {even }}(2)$ such that $\beta \psi \neq 0$ and $\operatorname{deg} \beta \bar{\psi}=2^{r}-2$ (see $(* *)$ ). Then $\alpha \mathrm{Sq}^{2^{s}} \psi \neq 0$ contradicts the maximality of $\operatorname{deg} \theta$. Q.E.D.

Corollary 8.8. $\alpha \mathrm{Sq}^{2^{s}} \neq 0$ if $2^{s} \notin 2 n$ and $2^{s+1} \in 2 n$.

Proof. Suppose $2^{s} \notin 2 n$ but $2^{s+1} \in 2 n$. By $(*), s=k_{i}$ where $1 \leqslant i \leqslant m$. If $i=m$, then $\theta=\mathrm{Sq}^{2^{s}} \theta^{\prime}$. If $i<m$, then $k_{i+1} \leqslant k_{i}-2$. So $\theta=\theta^{\prime} \mathrm{Sq}^{2^{s}} \theta^{\prime \prime}$, where $\theta^{\prime}$ is a monomial in $\left\{\mathrm{Sq}^{1}, \mathrm{Sq}^{2}, \ldots, \mathrm{Sq}^{2^{s-2}}\right\}$. By Rule 8.2, $\theta=\theta^{\prime} \mathrm{Sq}^{2^{s}} \boldsymbol{\theta}^{\prime \prime}=\mathrm{Sq}^{2^{s}} \boldsymbol{\theta}^{\prime} \boldsymbol{\theta}^{\prime \prime}$. Q.E.D. 
(B) Proof of Proposition 8.6(a). We will assume that Proposition 8.6 and, hence, Theorem 8.1 hold in even degree $<2 n$. We will prove Proposition $8.6(\mathrm{a})$ in degree $2 n$ by induction on $i$.

Initial case $i=1$. Write $\theta=\theta^{\prime} \mathrm{Sq}^{2^{t}}$. Since $\mathscr{A}(2)$ acts unstably on $H^{*}$ we have $t \leqslant r$. To eliminate $t<r$ observe that $\operatorname{deg} \alpha \theta^{\prime}=2^{r+1}+2^{t}-2$. If $t<r$ then, by Proposition 6.1, we have $\alpha \theta^{\prime} \mathrm{Sq}^{2 k} \neq 0$ for $2 k>2^{t}$. This contradicts the fact that $\theta$ is chosen to have maximal even degree so that $\alpha \theta \neq 0$.

General case. Assume 8.6(a) holds for $j \leqslant i$. So we can write

$$
\theta=\theta^{\prime} \mathrm{Sq}^{2^{t}} \theta_{r-i+1}^{k_{t}} \theta_{r-i+2}^{k_{i-1}} \cdots \theta_{r}^{k_{1}} \text {. }
$$

We want to show $t=r-i$.

First of all

$$
t \geqslant r-i
$$

Otherwise, by Rule 8.2,

$$
\boldsymbol{\alpha} \theta=\boldsymbol{\alpha} \boldsymbol{\theta}^{\prime} \mathrm{Sq}^{2^{\prime}} \boldsymbol{\theta}_{r-i+1}^{k_{i}} \cdots \theta_{r}^{k_{1}}=\alpha \boldsymbol{\theta}^{\prime} \theta_{r-i+1}^{k_{i}} \cdots \theta_{r}^{k_{1}} \mathrm{Sq}^{2^{\prime}} .
$$

Now $\operatorname{deg} \alpha \theta^{\prime} \theta_{r-i+1}^{k_{i}} \cdots \theta_{r}^{k}=2^{r+1}+2^{t}-2$. So, by Proposition 6.1, we have $\boldsymbol{\alpha} \boldsymbol{\theta}^{\prime} \theta_{r-i+1}^{k_{i}} \cdots \theta_{r}^{k_{1}} \mathrm{Sq}^{2 k} \neq 0$ for $2 k>2^{t}$. This contradicts the fact that $\theta$ was chosen to have maximal even degree and satisfy $\alpha \theta \neq 0$.

Second,

$$
t \leqslant r-i \text {. }
$$

Since $\theta$ is allowable, $t \leqslant k_{i}$. By Rule 8.5, $t<k_{i}$. Suppose $r-i<t<k_{i}$. By Rule 8.2 we can replace $\mathrm{Sq}^{2^{t}} \theta_{r-i+1}^{k_{i}}$ in $\theta$ by $\theta^{\prime \prime} \mathrm{Sq}^{2^{t}} \mathrm{Sq}^{2^{t+1}} \mathrm{Sq}^{2^{t}} \theta^{\prime \prime \prime}$. So

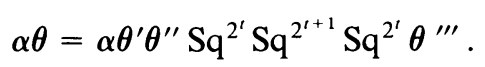

On the other hand, by Rule 8.4, we may replace $\mathrm{Sq}^{2^{t}} \mathrm{Sq}^{2^{t+1}} \mathrm{Sq}^{2^{t}}$ by $\mathrm{Sq}^{2^{t+1}} \mathrm{Sq}^{2^{t+1}}$. So

$$
\alpha \theta=\alpha \theta^{\prime} \theta^{\prime \prime} \mathrm{Sq}^{2^{t+1}} \mathrm{Sq}^{2^{t+1}} \theta^{\prime \prime \prime} .
$$

However $\alpha \theta^{\prime} \theta^{\prime \prime} \mathrm{Sq}^{2^{\prime}} \mathrm{Sq}^{2^{\prime+1}} \mathrm{Sq}^{2^{\prime}} \neq 0$ and $\theta^{\prime} \theta^{\prime \prime} \mathrm{Sq}^{2^{t+1}} \mathrm{Sq}^{2^{\prime+1}} \neq 0$ contradict the following restiction.

LEMmA 8.9. Either $\mathrm{Sq}^{2^{t+1}}$ or $\mathrm{Sq}^{2^{t+1}} \mathrm{Sq}^{2^{t}}$ act trivially on $\hat{Q}$ in any given degree $<2 n$ $+\left(2^{t+1}+2^{t}\right)$

Proof. We can assume that 8.6, and hence 8.1, holds in degree $<2 n$. If we dualize, then, given $x \in \hat{Q}^{2 k}$ where $2 k<2 n-\left(2^{t+1}+2^{t}\right), \mathrm{Sq}^{2^{t}} x \neq 0$ implies $2^{t} \in 2 k$ while $\mathrm{Sq}^{2^{t+1}} x \neq 0$ implies $2^{t+1} \in 2 k$. If we let $y=\operatorname{Sq}^{2^{t}} x$, then it follows from the above that $2^{t+1} \notin \operatorname{deg} y=2 k+2^{t}$. We are still in low enough degree to apply 8.1 and conclude

$$
\mathrm{Sq}^{2^{t+1}} \mathrm{Sq}^{2^{t}} x=\mathrm{Sq}^{2^{t+1}} x=0 . \quad \text { Q.E.D. }
$$

(C) Proof of Proposition 8.6(b). As in (B) we will assume that 8.1 holds in degree $<2 n$. We can also assume 8.6 (a) holds in degree $2 n$. Since $\theta$ is allowable, $k_{m} \leqslant k_{m-1} \leqslant \cdots \leqslant k_{1}$. Suppose $k_{i}<k_{i+1}<\cdots<k_{1}$. If $k_{i+1}=k_{i}(=t+1)$, then

$$
\alpha \theta=\alpha \theta^{\prime} \theta_{r-i}^{t+1} \theta_{r-i+1}^{t+1} \theta_{r-i+2}^{k_{i-1}} \cdots \theta_{r}^{k_{1}}
$$


where $k_{i-1} \geqslant t+2$. Use Rule 8.2. We have

$$
\begin{aligned}
\alpha \theta & =\alpha \theta^{\prime} \mathrm{Sq}^{2^{t+1}} \mathrm{Sq}^{2^{t}} \theta_{r-i}^{t-1} \mathrm{Sq}^{2^{t+1}} \theta_{r-i+1}^{t} \theta_{r-i+2}^{k_{1}+1} \cdots \theta_{r}^{k_{1}} \\
& =\alpha \theta^{\prime} \mathrm{Sq}^{2^{t+1}} \mathrm{Sq}^{2^{t}} \mathrm{Sq}^{2^{t+1}} \theta_{r-i}^{t-1} \theta_{r-i+1}^{t} \theta_{r-i+1}^{t} \theta_{r-i+2}^{k_{i-1}^{1}} \cdots \theta_{r}^{k_{1}} .
\end{aligned}
$$

By Rule 8.3 we can replace $\mathrm{Sq}^{2^{t+1}} \mathrm{Sq}^{2^{t}} \mathrm{Sq}^{2^{t+1}}$ by $\mathrm{Sq}^{2^{t+2}} \mathrm{Sq}^{2^{t}}+\mathrm{Sq}^{2^{t}} \mathrm{Sq}^{2^{t+2}}$. We now show that such a substitution forces $\alpha \theta=0$. Let $\bar{\alpha}=\alpha \theta^{\prime}$. We will show

(a) $\bar{\alpha} \mathrm{Sq}^{2^{t+2}} \mathrm{Sq}^{2^{t}}=0$

(b) $\bar{\alpha} \mathrm{Sq}^{2^{t}} \mathrm{Sq}^{2^{t+2}}=0$.

Let $2 k=\operatorname{deg} \bar{\alpha}$. Then

$$
2 k=2^{k_{1}+1}+\cdots+2^{k_{-1}+1}+2^{t+1}+2^{t}+2^{r-i}-2 .
$$

In particular $k_{i-1} \geqslant k_{i}=t+2$.

First, consider $\bar{\alpha} \mathrm{Sq}^{2^{t+2}} \mathrm{Sq}^{2^{t}}$. Let $2 l=\operatorname{deg} \bar{\alpha} \mathrm{Sq}^{2^{t+2}}$. Then $2 l=2 k-2^{t+2}$. So, $2^{t} \in$ $2 k$ forces $2^{t} \in 2 l$. By 8.1(a), $\alpha \mathrm{Sq}^{2^{t+2}} \mathrm{Sq}^{2^{t}}=0$.

Second, consider $\bar{\alpha} \mathrm{Sq}^{2^{t}} \mathrm{Sq}^{2^{t+2}}$. Let $2 l^{\prime}=\operatorname{deg} \bar{\alpha} \mathrm{Sq}^{2^{t}}$. Then $2 l^{\prime}=2 k-2^{t}$. So, by $(*), \lambda\left(2 l^{\prime}\right)=\lambda(2 k)-1=r-1$. By 8.1(b) we can find $\theta^{\prime \prime \prime}$ such that $\bar{\alpha} \theta^{\prime \prime \prime} \neq 0$, where $\operatorname{deg} \bar{\alpha} \theta^{\prime \prime \prime}=2^{r}-1$. But, then $\alpha \theta^{\prime} \mathrm{Sq}^{2^{t}} \theta^{\prime \prime \prime} \neq 0$ contradicts the fact that $\theta$ was chosen to have the maximal even degree such that $\alpha \theta \neq 0$.

\section{REFERENCES}

1. W. Browder, Torsion in H-spaces, Ann. of Math. (2) 74 (1961), 24-51.

2. Differential Hopf algebras, Trans. Amer. Math. Soc. 107 (1963), 153-176.

3. J. R. Hubbuck and R. Kane, On $\Pi_{3}$ of a finite H-space, Trans. Amer. Math. Soc. 213 (1975), 99-105.

4. R. Kane, On loop spaces without p torsion, Pacific J. Math. 60 (1975), 189-201.

5. The module of indecomposables for mod 2 finite H-spaces, Trans. Amer. Math. Soc. 249 (1979), 425-433.

6. Implications in Morava K-theory (to appear).

7. J. Lin. Torsion in H-spaces. I, Ann. of Math. (2) 103 (1976), 457-486.

8. , Even generators in the mod 2 cohomologv of a finite H-space, Math. Scand. 45 (1979), $295-312$.

9. H_ Higher order operations in the mod 2 cohomologv of a finite H-space, Amer. J. Math. 105 (1983), 855-937.

10. . Two torsion in the cohomology of finite H-spaces, J. Pure Appl. Math. 22 (1981), 265-292.

11. Two torsion and the loop space conjectures, Ann. of Math. (2) 115 (1982), 35-91.

12. J. Milnor, The Steenrod algebra and its dual, Ann. of Math. (2) 67 (1958), 150-171.

13. J. Milnor and J. C. Moore, On the structure of the Hopf algebras, Ann. of Math. (2) 81 (1965), 211-264.

14. E. Thomas, Steenrod squares and H-spaces, Ann. of Math. (2) 77 (1963), 306-317.

15. Steenrod squares and H-spaces. II, Ann. of Math. (2) 81 (1965), 473-495.

16. C. T. C. Wall, Generators and relations for the Steenrod algebra, Ann. of Math. (2) 72 (1960), $429-444$.

17. A. Zabrodsky, Secondary operations in the module of indecomposables, Algebraic Topology Conference, Aarhus, 1970.

Department of Mathematics, The University of Western Ontario, London, Ontario, Canada N6A 5B7 